\title{
Spike Afterpotentials Shape the In Vivo Burst Activity of Principal Cells in Medial Entorhinal Cortex
}

\author{
Dóra É. Csordás, Caroline Fischer, Johannes Nagele, ${ }^{\circledR}$ Martin Stemmler, and ${ }^{\circledR}$ Andreas V.M. Herz \\ Bernstein Center for Computational Neuroscience Munich and Faculty of Biology, Ludwig-Maximilians-Universität München, Martinsried-Planegg \\ 82152, Germany
}

Principal neurons in rodent medial entorhinal cortex (MEC) generate high-frequency bursts during natural behavior. While in vitro studies point to potential mechanisms that could support such burst sequences, it remains unclear whether these mechanisms are effective under in vivo conditions. In this study, we focused on the membrane-potential dynamics immediately following action potentials (APs), as measured in whole-cell recordings from male mice running in virtual corridors (Domnisoru et al., 2013). These afterpotentials consisted either of a hyperpolarization, an extended ramp-like shoulder, or a depolarization reminiscent of depolarizing afterpotentials (DAPs) recorded in vitro in MEC principal neurons. Next, we correlated the afterpotentials with the cells' propensity to fire bursts. All DAP cells with known location resided in Layer II, generated bursts, and their interspike intervals (ISIs) were typically between 5 and $15 \mathrm{~ms}$. The ISI distributions of Layer-II cells without DAPs peaked sharply at around $4 \mathrm{~ms}$ and varied only minimally across that group. This dichotomy in burst behavior is explained by cell-group-specific DAP dynamics. The same two groups of bursting neurons also emerged when we clustered extracellular spike-train autocorrelations measured in real 2D arenas (Latuske et al., 2015). Apart from slight variations in grid spacing, no difference in the spatial coding properties of the grid cells across all three groups was discernible. Layer III neurons were only sparsely bursting (SB) and had no DAPs. As various mechanisms for modulating ion-channels underlying DAPs exist, our results suggest that temporal features of MEC activity can be altered while maintaining the cells' overall spatial tuning characteristics.

Key words: bursts; grid cells; medial entorhinal cortex; spatial navigation; spike afterpotentials; whole-cell recording in vivo

Significance Statement

Depolarizing afterpotentials (DAPs) are frequently observed in principal neurons from slice preparations of rodent medial entorhinal cortex (MEC), but their functional role in vivo is unknown. Analyzing whole-cell data from mice running on virtual tracks, we show that DAPs do occur during behavior. Cells with prominent DAPs are found in Layer II; their interspike intervals (ISIs) reflect DAP time-scales. In contrast, neither the rarely bursting cells in Layer III, nor the high-frequency bursters in Layer II, have a DAP. Extracellular recordings from mice exploring real 2D arenas demonstrate that grid cells within these three groups have similar spatial coding properties. We conclude that DAPs shape the temporal response characteristics of principal neurons in MEC with little effect on spatial properties.

\section{Introduction}

Principal neurons in the superficial layers of medial entorhinal cortex (MEC) show rich temporal behavior, from slow depolarization ramps (Domnisoru et al., 2013; Schmidt-Hieber and

Received Oct. 28, 2019; revised Apr. 3, 2020; accepted Apr. 11, 2020.

Author contributions: D.É.C., C.F., J.N., M.S., and A.V.M.H. designed research; D.É.C., C.F., and J.N. performed research; D.É.C., C.F., and J.N. analyzed data; D.É.C., C.F., J.N., M.S., and A.V.M.H. wrote the paper.

D.É.C., C.F., and J.N. contributed equally to this work.

This work was supported by the German Federal Ministry for Education and Research Grant $01 G 00440$.

The authors declare no competing financial interests.

Correspondence should be addressed to Andreas V. M. Herz at herz@bio.Imu.de.

https://doi.org/10.1523/JNEUROSCI.2569-19.2020

Copyright $\odot 2020$ the authors
Häusser, 2013), spike locking and phase precession in the $\theta$ band (Hafting et al., 2008; Reifenstein et al., 2012), to $\gamma$-band activity (Chrobak and Buzsáki, 1998; Colgin et al., 2009) and burst sequences with instantaneous firing rates of up to $300 \mathrm{~Hz}$ (Latuske et al., 2015). The spatial firing fields of one particular MEC cell class, namely grid cells, form hexagonal lattices spanning the explored 2D environment (Hafting et al., 2005). Notably, not every grid cell participates to the same degree in these temporal phenomena. In particular, there are two subclasses of grid cells, those that burst frequently and those that do not or only rarely generate bursts (Mizuseki et al., 2009; Latuske et al., 2015; Ebbesen et al., 2016). But what is the mechanism behind the MEC bursts and what role do they play for spatially selective neurons, such as grid cells? 
In this study, we tested the hypothesis that burst activity of principal neurons in MEC is shaped by cell-intrinsic membranepotential dynamics. While extrinsic factors, such as $\theta$-band coupling in MEC Layer-II stellate cells change the likelihood of burst generation (Alonso and Klink, 1993; Engel et al., 2008; see also Hasselmo, 2014; Newman and Hasselmo, 2014) and the timing of spikes within a burst could reflect the arrival of strongly coincident synaptic input, we focus on the short-term dynamics that are triggered in the wake of an action potential (AP).

Indeed, slice experiments have shown that depolarizing afterpotentials (DAPs) arise in a majority of principle cells in superficial MEC layers (Alonso and Klink, 1993; Canto and Witter, 2012). DAPs are at the center of triphasic deflections following an AP, sandwiched between fast and medium after-hyperpolarization (fAHP and $\mathrm{mAHP}$ ). The DAP maximum occurs some 5$10 \mathrm{~ms}$ after the AP and peaks a few millivolts above the fAHP minimum. In stellate cells, DAPs become more pronounced when neurons are hyperpolarized, whereas the reverse is true for pyramidal neurons (Alessi et al., 2016). Not all cell types associated with grid-like rate maps have DAPs in vitro, however. In particular, Layer-III neurons are reported to have no DAPs (Canto and Witter, 2012).

DAPs do not only agree in their relevant time scale with intraburst interspike intervals (ISIs); in vitro, DAPs also play a causal role for bursting. Alessi et al. (2016) reported that during DAPs the AP current threshold was reduced such that the cells' average excitability increased by over $40 \%$. Conversely, neurons without strong DAPs did not burst at the beginning of an AP train (Canto and Witter, 2012).

To test the functional relevance of DAPs under in vivo conditions, we analyzed whole-cell recordings from mice moving on a virtual linear track (Domnisoru et al., 2013) and could show that DAPs play a decisive role for burst firing in MEC Layer-II neurons: Cells with DAPs were bursty and their intraburst ISIs were compatible with the DAP mechanism. ISI distributions of the other Layer-II cells were highly uniform and had a sharp peak at $4.1 \pm 0.2 \mathrm{~ms}$ (SD across this cell group). The remaining neurons were sparsely bursting (SB) and those with known location resided in Layer III, apart from one pyramidal cell in Layer II. The results are compatible with our findings for extracellular recordings from open-field arenas (Latuske et al., 2015). In addition, bursty cells with and without DAP did not differ in their spatial coding properties, apart from a slight change in grid spacing. As the ionic conductances that support DAPs are subject to modulatory factors, our analysis suggests that temporal features of grid-cell activity can be altered to serve different functions without affecting the cells' qualitative spatial tuning characteristics.

\section{Materials and Methods}

Data

We analyzed data from two separate studies in navigating wild-type (C57BL/6) male mice. Dataset D (Domnisoru et al., 2013) contained voltage traces from whole-cell recordings sampled at $20 \mathrm{kHz}$ in headfixed animals. These mice ran on cylindrical treadmills through virtual corridors. Dataset L (Latuske et al., 2015) contained tetrode data (sampling frequency: 20 or $24 \mathrm{kHz}$ ) obtained during movements in a real square arena $(70 \times 70 \mathrm{~cm})$.

\section{Cell selection}

\section{Dataset D}

The original dataset contained recordings from 51 cells of which 27 had been classified as grid cells by Domnisoru et al. (2013). One grid-cell recording was partially corrupted and excluded. Two grid cells had mean firing rates above $10 \mathrm{~Hz}$ and were removed to allow for an unbiased comparison with dataset $\mathrm{L}$, which contained only cells with firing rates below $10 \mathrm{~Hz}$ to exclude interneurons. From the 24 neurons that had been classified as non-grid cells two cells had firing rates above $10 \mathrm{~Hz}$ and the APs of six other cells did not meet our criteria (see below, Membrane-potential dynamics). This resulted in 40 neurons from dataset $\mathrm{D}$, namely 24 grid cells and 16 non-grid cells.

\section{Dataset $L$}

After removing cells for which the animal trajectories showed artifacts, 522 principal cells were identified using the same criterium (mean firing rate $<10 \mathrm{~Hz}$ ) as in Latuske et al. (2015). Out of those cells, 115 cells had been classified as grid cells by these authors. Ten of the 522 cells were not considered further as their ISI distributions differed strongly from all other cells in that they had not a single ISI below $8 \mathrm{~ms}$. Similarly, to avoid artifacts in the cluster analysis of the cells' spike-time autocorrelations, seven cells with sparse autocorrelations were removed (see below for details). Altogether, this led to 505 cells in dataset L, 112 grid cells and 393 non-grid cells.

\section{Spike-train characterization}

The firing rate of a cell was defined as number of spikes divided by the total duration of the recording. For the graphical illustrations, spiketime autocorrelations and ISI distributions were calculated from binned data (bin width: $1 \mathrm{~ms}$ ). To compute the peak location and width of ISI distributions, the recorded time difference between each pair of successive spikes was represented by a Gaussian kernel with a SD of $1 \mathrm{~ms}$, centered at the measured time difference. These individual kernel density (KD) estimates were summed up across the entire recording. The analogous procedure was used for autocorrelations.

The location of the ISI peak was determined as the ISI for which the KD estimate was maximal. Similarly, the width of the ISI distribution was defined as full width at half maximum. The mean ISI and its SD were calculated from all ISIs, the coefficient of variation (CV) was defined as the ratio of SD to mean.

A burst was defined as a sequence of at least two spikes with ISIs shorter than $8 \mathrm{~ms}$. The fraction of ISIs smaller than $8 \mathrm{~ms}$ was calculated relative to all ISIs and serves as a measure for the cell's burstiness. An event is a burst or an isolated spike. The fraction of single spikes was defined as the number of spikes that do not belong to a burst divided by the number of all events.

\section{Principal-component analysis (PCA)}

For both datasets, autocorrelations were calculated for time lags between $0 \mathrm{~ms}$ and $\tau_{\max }=50 \mathrm{~ms}$. To weigh all neurons equally autocorrelations were normalized to unit area. Principal components were calculated after binning (bin width: $1 \mathrm{~ms}$ ). To reduce spurious effects caused by sparse normalized autocorrelations, cells with $>75 \%$ empty bins (the maximum value for dataset $\mathrm{D}$ ) were removed (one grid cell and six nongrid cells in dataset L). For the same reason, five (two) cells of dataset $\mathrm{D}$ (dataset L) that had relatively few spikes $(<130)$ were excluded when PC components were calculated but are included in the further analysis. To test the robustness of the PCA of the D data, the maximal time lag $\tau_{\max }$ was varied between 30 and $100 \mathrm{~ms}$ (see also Results).

\section{Identification of neuron classes}

For the $\mathrm{D}$ dataset, visual inspection of the $2 \mathrm{D}$ space spanned by the first two PCs suggested two main cell groups, whose arrangement was determined by $\mathrm{k}$-means clustering with $\mathrm{k}=2$ clusters. To test the robustness of the k-means clustering for the $\mathrm{L}$ dataset, cluster analyses were performed on the 50-dimensional binned autocorrelations as well as in PC spaces with $N=2-4$ dimensions. The clustering quality was estimated through silhouette scores (Rousseeuw, 1987).

\section{Membrane-potential dynamics}

The whole-cell voltage traces contained sizeable fluctuations that reflected synaptic inputs and potential movement artefacts. To obtain reliable information about the membrane potential before and after an AP, AP-triggered averaging was performed. The APs themselves varied in amplitude and width, both within and across the different recordings, 
suggesting that the recording quality varied in time; the slowly decaying AP amplitudes and increasing width of some cells indicated run-down effects. To guarantee a good recording quality and to obtain reliable estimates of the subthreshold membrane-potential dynamics on the time scales relevant for fAHPs and DAPs, we focused on well isolated APs (no further APs within $25 \mathrm{~ms}$ before and after the trigger $\mathrm{AP}$ ), and required the individual AP amplitudes to be larger than $40 \mathrm{mV}$ (measured relative to the membrane potential $10 \mathrm{~ms}$ before the AP maximum) and APs width (full width at half height) to be smaller than $1 \mathrm{~ms}$.

The pre-AP voltage slope was calculated from the cell's average AP-triggered voltage trace within the last $10 \mathrm{~ms}$ before AP onset; AP onset was determined by a threshold crossing $(15 \mathrm{mV} / \mathrm{ms})$ in the first derivative of the average AP-triggered voltage trace. For single APs, the AP threshold was determined by the point of maximal curvature in the voltage trace (after Gaussian-filtering the voltage trace with sigma $=0.1 \mathrm{~ms}$ ).

For the intraburst voltage traces shown in Figure $1 C$, we collected all first and second APs (amplitude $>40 \mathrm{mV}$, width $<1 \mathrm{~ms}$ ) within a burst, i.e., all APs following an ISI longer than $8 \mathrm{~ms}$ and followed by an ISI shorter than $8 \mathrm{~ms}$ ("first APs") and all APs following a first AP with an ISI shorter than $8 \mathrm{~ms}$ ("second APs"), respectively. To illustrate the time evolution of the membrane potential, we either aligned the voltage traces at the first AP and cut the trace when the threshold for the second AP was reached (Fig. 1C, left panels) or aligned the traces at the second AP (Fig. 1C, right panels). In that case, the highlighted traces start at two AP-widths after the first AP and end at the threshold crossing of the third AP in the burst or $8 \mathrm{~ms}$ after the second AP, whichever comes first. Mean voltage traces (Fig. 1C, black lines) are calculated form these truncated samples to minimize influences by AP upstrokes or downstrokes.

For cells with DAPs, the fAHP amplitude $\Delta \mathrm{V}_{\mathrm{fAHP}}$ was defined as the average voltage minimum during the fAHP relative to the voltage at $\mathrm{AP}$ onset. This means that $\Delta \mathrm{V}_{\mathrm{fAHP}}$ is negative for DAP cells (Fig. 1). The DAP-deflection $\Delta V_{\text {DAP }}$ was defined as the difference between the voltage level at the DAP peak and at the minimum of the preceding fAHP. It is positive for cells with DAPs. The time interval between the AP peak and the following fAHP minimum is denoted by $\Delta \mathrm{t}_{\mathrm{fAHP}}$, the time interval between the AP peak and the following DAP maximum is called $\Delta t_{D A P}$.

To compare the after potentials of all recorded neurons, the definitions of $\Delta \mathrm{V}_{\mathrm{fAHP}}$ and $\Delta \mathrm{V}_{\mathrm{DAP}}$ had to be generalized to cells without DAP. This was done (1) for grid cells only, and (2) for all neurons. To this end, we calculated the population averages $-\Delta t_{f A H P}$ and $-\Delta t_{D A P}$ across the respective DAP cells (grid cells: $n=8$, all neurons: $n=15$ ). We then used these mean time intervals (grid cells: $\Delta t_{1} \equiv-\Delta t_{f A H P}=1.8 \pm$ $0.4 \mathrm{~ms}, \quad \Delta t_{2} \equiv-\Delta t_{D A P}=4.6 \pm 1.2 \mathrm{~ms}$, all neurons: $\Delta t_{1} \equiv-\Delta t_{f A H P}=2.0 \pm 0.5 \mathrm{~ms}, \Delta t_{2} \equiv-\Delta t_{D A P}=5.4 \pm$ $2.3 \mathrm{~ms}$ ) to determine voltage changes corresponding to $\Delta \mathrm{V}_{\mathrm{fAHP}}$ and $\Delta \mathrm{V}_{\mathrm{DAP}}$ for cells without DAP. These are called $\Delta \mathrm{V}_{1}$ and $\Delta \mathrm{V}_{2}$, respectively. For notational simplicity, we will use these terms for DAP cells, too, but here, they denote the $\Delta \mathrm{V}_{\mathrm{fAHP}}$ and $\Delta \mathrm{V}_{\mathrm{DAP}}$ values measured at the cell-specific $\Delta \mathrm{t}_{\mathrm{fAHP}}$ and $\Delta \mathrm{t}_{\mathrm{DAP}}$ values.

To characterize the subthreshold behavior following the first AP within a burst, we determined $\Delta \mathrm{V}_{1}$ and $\Delta \mathrm{V}_{2}$ values from the traces between the first and second $\mathrm{AP}$ and used the cell-specific $\Delta t_{1}$

D
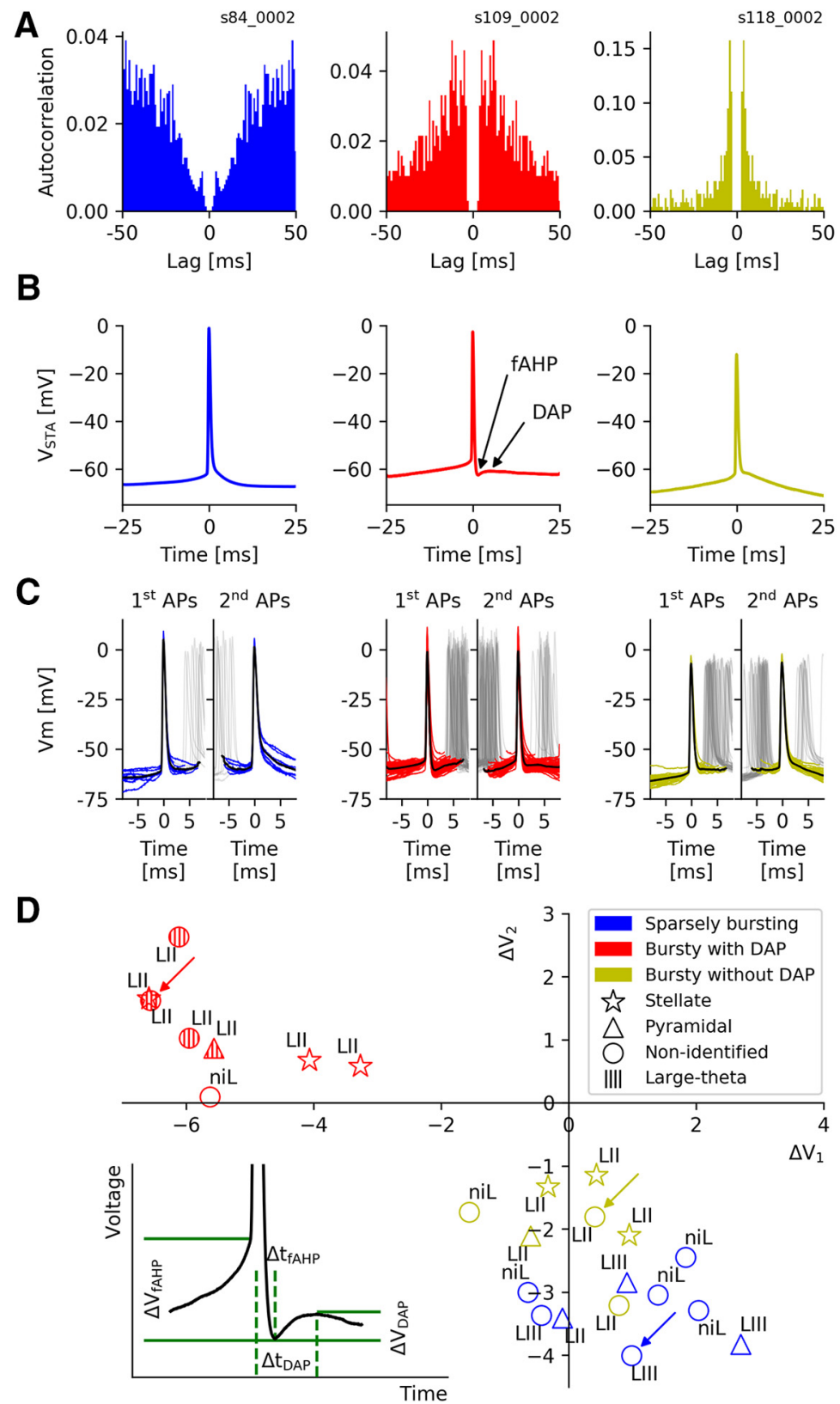

Figure 1. Spike afterpotentials of MEC grid cells from mice moving in virtual corridors. $A$, Typical examples of grid-cell burst behavior. Left panel, Autocorrelation function of a SB cell. Middle panel, A bursting cell with broad autocorrelation flanks. Right panel, A bursting cell with sharply peaked autocorrelation. Note the different scale on the $y$-axis. $\boldsymbol{B}$, Grid cells differ in their spike afterpotentials, as shown by the spike-triggered averages of the voltage traces of isolated APs (no further APs within $25 \mathrm{~ms}$ before and after the trigger AP). Left panel, A monotone repolarization that is gradually slowing down. Middle panel, fAHP followed by a DAP. Right panel, A short repolarization that abruptly turns into a much slower voltage decay, which may include an initial flat shoulder. $C$, These dynamics occur during bursts, too, as shown by voltage traces around the first and second AP in a burst (left and right subpanels, respectively). The fAHP/DAP complex is more pronounced after the first spike (middle panel), and the membrane potential decreases more rapidly after the second spike in the two example cells without fAHP/DAP complex, in agreement with the lack (left panel) or reduction (right panel) of further spikes in the burst. $\boldsymbol{D}$, Characterization of spike afterpotentials. Inset, Definition of parameters. Main panel, Group data. To characterize afterpotentials for cells without DAPs, the two parameters $\Delta \mathrm{V}_{1}$ and $\Delta \mathrm{V}_{2}$ take the role of $\Delta \mathrm{V}_{\text {fAHP }}$ and $\Delta V_{\text {DAP. }}$. The new parameters are determined in the same way as $\Delta V_{\text {fAHP }}$ and $\Delta V_{\text {DAP }}$, at times $\Delta t_{1}$ and $\Delta t_{2}$, respectively. These times are obtained from averages of $\Delta \mathrm{t}_{\mathrm{fAHP}}$ and $\Delta \mathrm{t}_{\mathrm{DAP}}$ across the population of DAP cells. To simplify the notation, the terms $\Delta \mathrm{V}_{1}$ and $\Delta \mathrm{V}_{2}$ (instead of $\Delta \mathrm{V}_{\text {fAHP }}$ and $\Delta \mathrm{V}_{\text {DAP }}$ ) are used for DAP cells, too.

and $\Delta t_{2}$ values calculated for isolated APs. For one DAP cell, $\Delta \mathrm{t}_{1}$ exceeded $8 \mathrm{~ms}$ so that this analysis could not be applied. Similarly, the second APs of six other cells did not meet the AP-width criterium so that in total, seven cells had to be excluded. 


\section{Spatial coding properties}

For dataset L, firing rate maps were computed as in Latuske et al. (2015). Based on these maps and their spatial autocorrelations, grid spacings were computed; grid scores and head-direction scores were calculated as in Sargolini et al. (2006), the spatial information as in Skaggs et al. (1996).

\section{Experimental design and statistical analysis}

We analyzed data recorded by Domnisoru et al. (2013) and Latuske et al. (2015) and refer the reader to these two publications for details on the experimental design. All our analyses were performed in Python 2.7.6. Specific statistical tests used are stated throughout the text. The KruskalWallis test, the Kolmogorov-Smirnov test, the $\chi^{2}$ test, and the median tests are taken from scipy.stats (0.17.0, RRID: SCR_008058). For an overview over SciPy, see Virtanen et al. (2020). The linear regression, the PCA and the k-means clustering are taken from scikit-learn (0.19.2, RRID: SCR_002577; Pedregosa et al., 2011). Unless noted otherwise, all errors are reported as SDs.

\section{Bootstrapping}

To assess the fAHP and DAP parameters, we bootstrapped the AP-triggered voltage traces of a cell by using sampling with replacement and repeated this procedure 10,000 times to obtain mean values and standard errors.

\section{Results}

The temporal firing characteristics of principal neurons in the MEC of behaving rodents vary strongly from cell to cell, even if their mean firing rates are almost identical (Latuske et al., 2015). Some neurons rarely fire with ISIs shorter than $8 \mathrm{~ms}$; their spiketime autocorrelations have a pronounced dip at short time lags (Fig. 1A, left). In other cells, the autocorrelation peaks in the 5to 15-ms range with broad flanks (Fig. 1A, middle); yet other cells have distinct autocorrelations that are sharply peaked at even shorter lags (Fig. $1 A$, right). The second and third group of neurons were collectively termed "bursty" by Latuske et al. (2015), who did not distinguish between the two groups, whereas the first group has been called "non-bursty" by these authors. Since non-bursty neurons generate bursts from time to time, too, we will call them "sparsely bursting" (SB), in line with Simonnet and Brecht (2019).

We wondered whether differences in the in vivo spike patterns of bursty neurons could be explained at a mechanistic level by differences in their intrinsic single-cell dynamics and whether, for the spatially tuned cells within the total population, differences in the cells' temporal discharge patterns were reflected in their spatial tuning properties. To this end, we analyzed wholecell recordings from mice moving on a linear track in virtual reality (Domnisoru et al., 2013) and extracellular recordings from mice navigating in 2D environments (Latuske et al., 2015).

We start with an analysis of grid cells and then extend our approach to non-grid cells. Before doing so, we note that recent work shows that true grid-cell firing in a virtual environment is only seen if the animal is free to rotate its head in a virtual environment (Chen et al., 2019). For this reason, when we use the term "grid cells" for the cells recorded by (Domnisoru et al., 2013) under head fixation, such a cell should be understood as a "putative grid cell."

\section{Grid cells differ in the voltage deflections following an AP}

We first focus on the intracellular linear-track data as these provide information about both spike times and membrane-potential dynamics.
The time courses of the membrane potentials recorded by Domnisoru et al. (2013) show striking cell-to-cell differences within the first $10 \mathrm{~ms}$ following an AP. Three distinct types of behavior can be distinguished from the spike-triggered voltage traces of isolated APs (such that no other APs occurred within $25 \mathrm{~ms}$ before or after the trigger AP): (1) a monotone repolarization that gradually slows down (Fig. $1 B$, left panel); (2) a fAHP followed by a DAP (Fig. $1 B$, middle panel); and (3) a short repolarizing phase that abruptly turns into a much slower voltage decay, which may include a flat shoulder (Fig. $1 B$, right panel).

Are these characteristic features only expressed when a cell fires isolated APs or can they also be observed during episodes of high spike activity? To answer this question, we analyzed bursts (Fig. 1C), defined as AP sequences with intraburst ISIs of $<8 \mathrm{~ms}$. Distinguishing between the voltage traces around the versus second AP in a burst (Fig. $1 C$, left vs right subpanels), shows that in cells with a fAHP/DAP complex, this voltage excursion is more pronounced after the first spike (Fig. $1 C$, middle panel). In the two cells without fAHP/DAP complex, the membrane potential decreases more rapidly after the second spike, in agreement with the lack (Fig. 1C, left panel) or strongly reduced number (Fig. $1 C$, right panel) of further APs in the burst.

To quantify these distinct behaviors, we used parameters that capture two salient features of cells exhibiting DAPs, the voltage minimum during the fAHP and the voltage peak during the DAP (Fig. $1 D$, lower left inset). The "fAHP-depth" $\Delta V_{\text {fAHP }}$ measures the voltage minimum relative to the membrane potential at AP onset. This minimum occurs at some time $\Delta \mathrm{t}_{\mathrm{fAHP}}$ after the AP peak. The "DAP-deflection" $\Delta \mathrm{V}_{\mathrm{DAP}}$ measures the difference between the membrane potential at the DAP peak and the fAHP minimum. The DAP peak is attained at some time $\Delta t_{D A P}$ after the AP peak (Fig. 1D).

To extend the $\Delta \mathrm{V}_{\mathrm{fAHP}}$ and $\Delta \mathrm{V}_{\mathrm{DAP}}$ measures to voltage traces of cells with no detectable DAP, two time intervals corresponding to $\Delta \mathrm{t}_{\mathrm{fAHP}}$ and $\Delta \mathrm{t}_{\mathrm{DAP}}$ need to be defined. For concreteness, we used the population means across all grid cells with a DAP $(n=8)$, resulting in $\Delta t_{1} \equiv-\Delta t_{f A H P}=1.8 \pm 0.4 \mathrm{~ms}$ and $\Delta t_{2} \equiv-\Delta t_{D A P}=4.6 \pm 1.2 \mathrm{~ms}$. We then determined the voltage differences corresponding to $\Delta \mathrm{V}_{\mathrm{fAHP}}$ and $\Delta \mathrm{V}_{\mathrm{DAP}}$ at these two time points, and named them $\Delta \mathrm{V}_{1}$ and $\Delta \mathrm{V}_{2}$, respectively. For DAP cells, we define $\Delta \mathrm{V}_{1}=\Delta \mathrm{V}_{\mathrm{fAHP}}$ and $\Delta \mathrm{V}_{2}=$ $\Delta \mathrm{V}_{\text {DAP }}$ to simplify the notation. These settings mean that cells with a shoulder or slow voltage decay (Fig. $1 B$, right panel) have zero or small $\Delta \mathrm{V}_{2}$ regardless of their $\Delta \mathrm{V}_{1}$ value, whereas large negative $\Delta \mathrm{V}_{2}$ values indicate a strong decline in membrane potential until around $5 \mathrm{~ms}$ after the AP.

Within the parameter space spanned by $\Delta \mathrm{V}_{1}$ and $\Delta \mathrm{V}_{2}$, neurons fall into two distinct groups (Fig. $1 D$ ), cells with a pronounced DAP (negative $\Delta V_{1}$ and positive $\Delta V_{2}$ ) and cells with no detectable DAP (negative $\Delta \mathrm{V}_{2}$ ), which typically have also no fAHP (positive $\Delta \mathrm{V}_{1}$ ). Results from a bootstrapping analysis (see Materials and Methods) underscore the reliability of the $\Delta \mathrm{V}_{1}$ and $\Delta V_{2}$ estimates (Fig. $2 A$ ). Whether we measured APs within bursts or isolated APs did not qualitatively change the scatter of data points in the $\Delta V_{1}-\Delta V_{2}$ plane nor the relation between the cell groups (Fig. $2 B$ ).

All measured neurons that had a DAP and whose location was known resided in Layer II. Five of these eight cells had large $\theta$-band membrane potential oscillations and have been called "large $\theta$ cells" by Domnisoru et al. (2013). Cells without a DAP $(n=16)$ were located in both Layers II and III. 
A

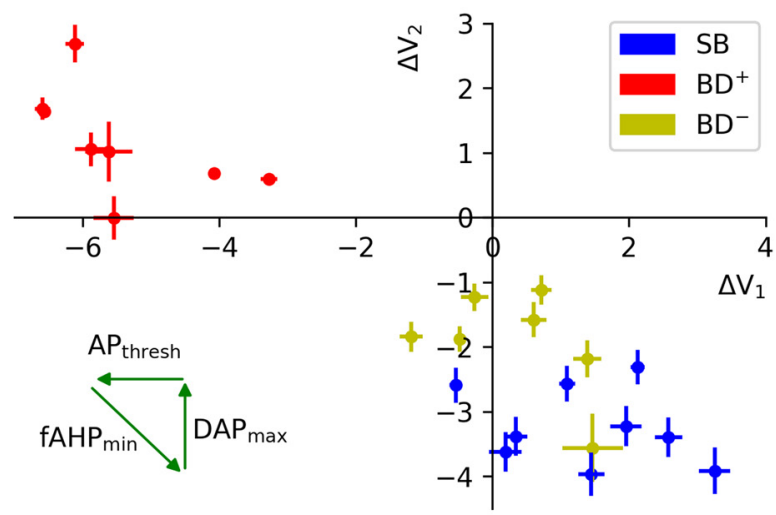

B

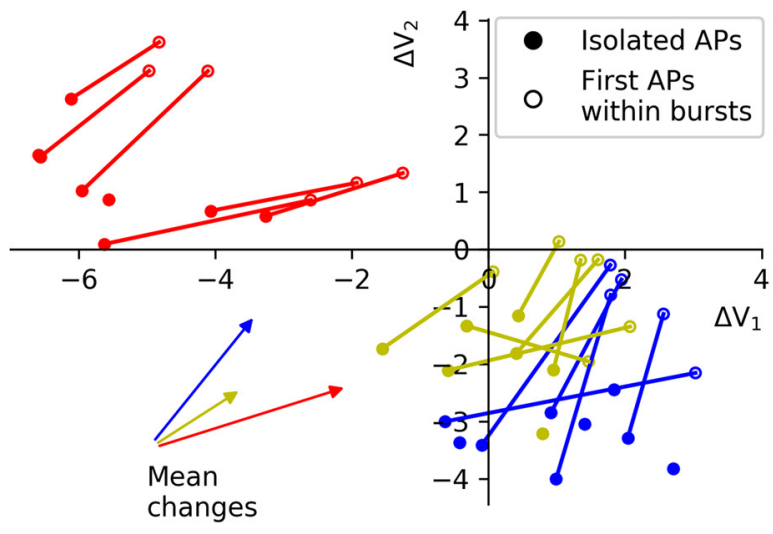

Figure 2. Stability of afterpotential parameters. $\boldsymbol{A}$, To quantify the reliability of the parameters characterizing spike afterpotentials, we conducted a bootstrapping analysis, using data from isolated APs. Error bars indicate SEM as obtained from 1000 samples and demonstrate that the fAHP and DAP parameters can be estimated reliably. The three arrows show how a cell's position in the $\Delta V_{1}-\Delta V_{2}$ space changes when the respective parameter is increased. $B, A s$ an additional test, we computed the parameters $\Delta \mathrm{V}_{1}$ and $\Delta \mathrm{V}_{2}$ from voltage traces following the first AP in a burst. With our criteria for APs, this was possible for 17 cells; the other cells are shown as isolated dots. For technical details, see Materials and Methods. For all but two cells, for which $\Delta \mathrm{V}_{1}$ and $\Delta \mathrm{V}_{2}$ could be determined for isolated APs and first APs within a burst, both quantities increase when computed from traces within a burst, as can also been seen from the mean changes in each cell class. Importantly, the original grouping in different cell classes is not affected.

\section{Grid cells differ in their spike-train characteristics}

To capture the diversity of spike discharge patterns we conducted a PCA on the spike-time autocorrelations of the 24 intracellularly recorded grid cells (Fig. $3 A$ ), as has been done for extracellular recordings (Latuske et al., 2015). We restricted our attention to autocorrelations on short time-scales, in particular to the region between 0 and $50 \mathrm{~ms}$ after a spike. We found that the first two principal components, PC1 and PC2, explain $66 \%$ and $18 \%$ of the cell-to-cell variability, respectively, whereas the contribution from PC3 adds only another $4 \%$. Together, PC1 and PC2 thus account for $84 \%$ of the variability. This value changes by $<3 \%$ when $\tau_{\max }$ is varied between 30 and $100 \mathrm{~ms}$ (data not shown) and starts to decrease for shorter or longer maximal lag. These findings indicate that a 2D PC representation of the grid-cell autocorrelations in the 0 - to 50-ms range describes the essence of the cell-to-cell variability.

The mean autocorrelation is highly peaked at a lag $\tau$ of around $4 \mathrm{~ms}$ (Fig. $3 B$ ), and so are both principal components (Fig. 3C). This indicates that brief activity bursts in the $250-\mathrm{Hz}$ range play an important role for both the mean grid-cell discharge patterns and their cell-to-cell variability.
A
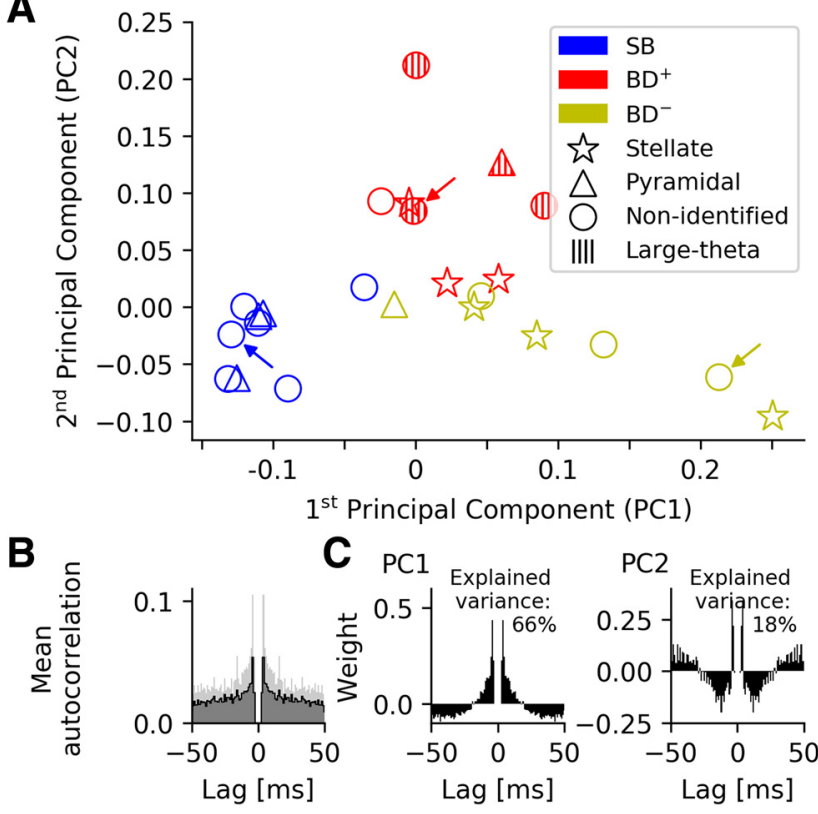

Figure 3. Spike-time autocorrelations of MEC grid cells from mice moving in virtual corridors. $\boldsymbol{A}$, visual inspection of spike-time autocorrelations suggests a separation in two major groups, SB and bursting. Based on the intracellular measurements (see Fig. 1), the group of bursting neurons can be subdivided in cells with $\mathrm{DAP}\left(\mathrm{BD}^{+}\right)$, which are shown in red, and cells without detectable DAP $\left(\mathrm{BD}^{-}\right)$, shown in yellow. The arrows mark the example neurons shown in Figure 1. One cell has intermediate properties and is assigned to the sparsely-bursting group (blue) by k-means clustering $(\mathrm{k}=2)$. $\boldsymbol{B}$, Mean autocorrelation. Shaded areas show the variability in the data (SD). $C$, The first two principal components of the spike-time autocorrelations. The pronounced peaks in $\boldsymbol{B}, \boldsymbol{C}$ demonstrate that ISIs of around $4 \mathrm{~ms}$ are indicative of both the mean grid-cell discharge patterns and their cell-to-cell variability.

Within this $2 \mathrm{D}$ representation (Fig. $3 A$ ), neurons without a DAP, shown in blue and yellow, have a negative or only small positive second principal component and strongly vary in their first principal component. Cells with large negative PC1 are SB as the example in the left panel of Figure $1 A$, whose position in the PC1/PC2 space is marked with a blue arrow in Figure $3 A$. Cells with positive PC1 burst like the cell in Figure $1 A$, right panel (Fig. 3A, yellow arrow). Cells with a DAP have positive PC2, only a small PC1, and are also bursting, although with a much broader peak in their autocorrelation as demonstrated by the example in Figure $1 A$, middle panel (Fig. $3 A$, red arrow).

This grouping is based on visual inspection of the AC principal components and might not properly distinguish between bursting and sparsely-bursting neurons with small PC1. To better discriminate between these two cell groups, we conducted a $\mathrm{k}$-means clustering with $\mathrm{k}=2$. The analysis suggested that nine cells should be classified as SB neurons; based on their intracellular characteristics, the remaining 15 bursting ("B") cells are either DAP cells ("BD" ${ }^{+}$) or cells without detectable DAP ("BD"”). The same clusters emerge if the spike data from the first and second half of each experiment are treated separately (data not shown), and provide evidence for the robustness of our approach.

All bursty neurons whose anatomic position was classified by Domnisoru et al., are located in Layer II, none in Layer III (two bursty cells were not assigned to a layer). Furthermore, unlike suggested by reports from rats (Ebbesen et al., 2016), bursty neurons are more likely to be stellate than pyramidal cells (six vs two cells), in agreement with the larger abundance of stellate cells compared with pyramidal cells (Alonso and Klink, 1993). There was no detectable difference in the morphology of bursty 
A

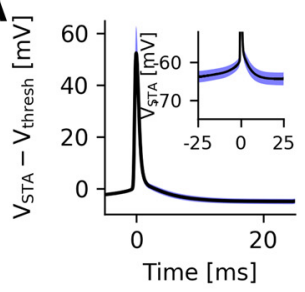

B
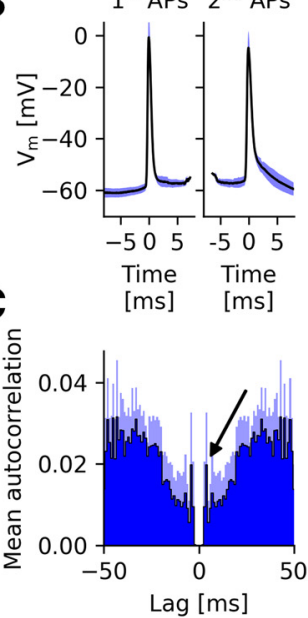

D

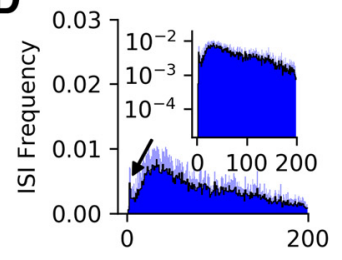

E

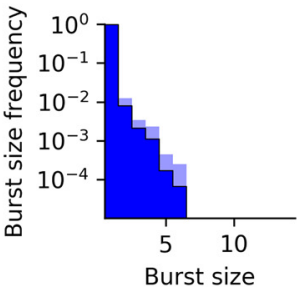

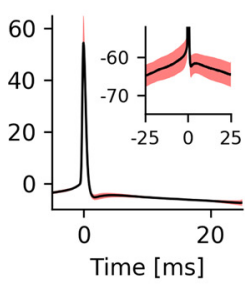
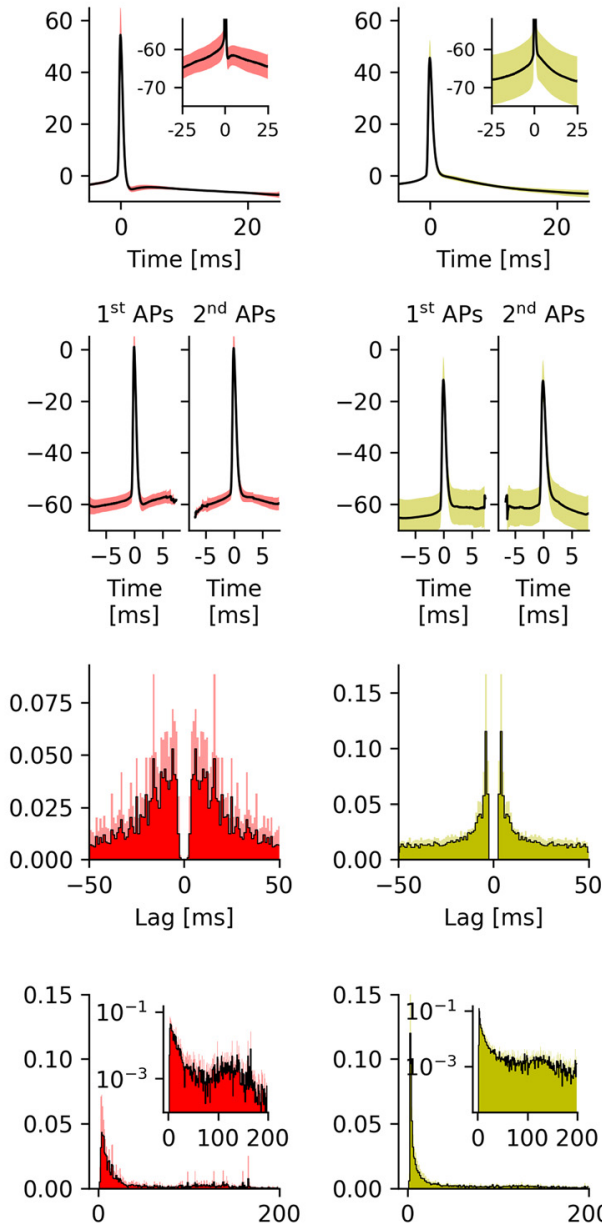

ISI [ms]

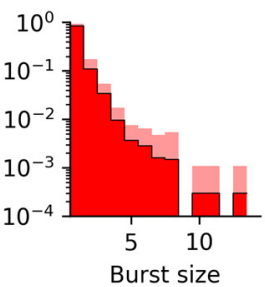

group of bursty neurons are correlated with a slope of -0.49 (SE: 0.06). The three groupings are robust, as confirmed by bootstrapping and indicated in Figure $2 A$, error bars.

Post-AP dynamics explain the spiketrain characteristics of bursty grid cells After dividing the cells into three groups based on their spike-train autocorrelations and DAP characteristics, we compared the group averages for the grid cells' intracellular voltage traces (Fig. 4). Confirming the impression from individual cells, sparsely-bursting neurons show a smooth and monotone AP down-stroke (Fig. 4A, left panel), bursty cells with DAP exhibit a local voltage minimum followed by repolarization (Fig. $4 A$, middle panel), and compared with SB cells, bursty cells without DAP tend to have two phases of repolarization: an initial AP downstroke followed abruptly by a slower rate of repolarization, yielding a kink in the voltage traces (Fig. $4 A$, right panel). Within bursts (Fig. 4B), the characteristic differences between the three cell classes are also apparent, with the voltage trace following the second AP in a burst decreasing more rapidly than the trace following the first $\mathrm{AP}$ for $\mathrm{SB}$ and $\mathrm{BD}^{-}$cells; for $\mathrm{BD}^{+}$cells, the second $\mathrm{AP}$ in a burst is generally not followed by a DAP, which could be a sign that the DAP mechanism benefits from a longer subthreshold period before the AP.

To visualize these distinct features, the spike-triggered voltage traces were averaged for each neuron and then aligned to the cell's mean voltage at AP onset. Without voltage alignment (see insets), group-to-group differences in membrane potential at the onset of isolated APs are apparent (SB: $-59.42 \pm$ $1.22 \mathrm{mV}, \mathrm{BD}^{+}:-57.12 \pm 3.09 \mathrm{mV}, \mathrm{BD}^{-}$: $-61.40 \pm 6.83 \mathrm{mV}$ ) but not significant $[p$ $\left(\mathrm{BD}^{+}, \mathrm{BD}^{-}\right)=0.25 ; p\left(\mathrm{BD}^{-}, \mathrm{SB}\right)=0.15 ; p$ $\left(\mathrm{BD}^{-}, \mathrm{SB}\right)=0.96$, Kruskal-Wallis test] , as the AP onset voltage varies throughout the experiment, including for the two groups of bursting neurons. We, therefore, focused on voltage slopes and voltage differences between isolated APs and APs occurring with bursts.

neurons with and without DAP: Within the $\mathrm{BD}^{+}$group $(n=8)$, there were three stellate cells, one pyramidal neuron and four non-identified cells. Within the $\mathrm{BD}^{-}$group $(n=7)$, there were three stellate cells, one pyramidal neuron and three non-identified cells. In contrast, not a single non-bursty cell was identified as a stellate cell (pyramidal and non-identified cells: three and six out of $n=9$, respectively) and non-bursty neurons may have a tendency to reside in Layer III (three vs one cell in Layer II; five cells were not classified). Finally, in the $\Delta \mathrm{V}_{1}-\Delta \mathrm{V}_{2}$ representation (Fig. 1D), $\mathrm{BD}^{-}$neurons overlap with $\mathrm{SB}$ cells, but tend to have less negative $\Delta \mathrm{V}_{2}$ values and the $\Delta \mathrm{V}_{1}$ and $\Delta \mathrm{V}_{2}$ values within the
The voltage slope during the last $10 \mathrm{~ms}$ before the onset of isolated APs does not differ significantly between $\mathrm{BD}^{+}$and $\mathrm{BD}^{-}$ neurons $\left(\mathrm{BD}^{+}: 0.50 \pm 0.03 \mathrm{mV} / \mathrm{ms} ; \mathrm{BD}^{-}: 0.48 \pm 0.08 \mathrm{mV} / \mathrm{ms}\right.$; $p=0.35$, Kruskal-Wallis test) but does so when bursty and sparsely-bursting neurons are compared (B: $0.49 \pm 0.06 \mathrm{mV} / \mathrm{ms}$; SB: $0.34 \pm 0.05 \mathrm{mV} / \mathrm{ms} ; p=0.00,015$, Kruskal-Wallis test). For all cell groups, the second AP in a burst was triggered at a higher voltage than the first $\mathrm{AP}\left[\mathrm{SB}: 2.51 \pm 0.57 \mathrm{mV}, \mathrm{BD}^{+}: 0.78 \pm\right.$ $0.72 \mathrm{mV}, \mathrm{BD}^{-}: 2.21 \pm 0.54 \mathrm{mV}$, with $p\left(\mathrm{BD}^{+}, \mathrm{BD}^{-}\right)=9.8 \times 10^{-3}$, $p\left(\mathrm{BD}^{+}, \mathrm{SB}\right)=3.4 \times 10^{-3}, p\left(\mathrm{BD}^{-}, \mathrm{SB}\right)=0.85$, Kruskal-Wallis 
test]. Isolated APs are triggered at voltages slightly lower than those of the first $\mathrm{AP}$ in a burst $\left(\mathrm{SB}:-0.96 \pm 0.60 \mathrm{mV}, \mathrm{BD}^{+}\right.$: $\left.-0.51 \pm 0.71 \mathrm{mV}, \mathrm{BD}^{-}:-0.36 \pm 0.63 \mathrm{mV}\right)$. These values do not differ significantly across the three cell groups $\left[p\left(\mathrm{BD}^{+}, \mathrm{BD}^{-}\right)=\right.$ $0.70 ; p\left(\mathrm{BD}^{+}, \mathrm{SB}\right)=0.38 ; p\left(\mathrm{BD}^{-}, \mathrm{SB}\right)=0.36$, Kruskal-Wallis test $]$. Finally, for $\mathrm{BD}^{+}$cells, the maximal depolarization reached during the DAP was highly variable; when a second AP was not triggered, the DAP maximum lay $3.75 \pm 0.63 \mathrm{mV}$ below the second AP's threshold, when averaged across cells. For each cell, the broad distribution of DAP maxima overlapped with the distribution of thresholds for the second AP, consistent with the hypothesis that DAPs play a role in burst firing of $\mathrm{BD}^{+}$cells.

The averaged autocorrelations (Fig. 4C, left panel) and ISIs (Fig. 4D, left panel) of sparsely-bursting cells show that although these neurons rarely generate spike sequences with short ISIs, only $2 \%$ of all their ISIs are $<8 \mathrm{~ms}$, if they do fire such bursts, there is a pronounced short ISI that is only $4.30 \pm 0.81 \mathrm{~ms}$ long (Fig. 4C,D, black arrows). Both types of bursty neurons exhibit prominent ISI- and autocorrelation peaks at short time scales (Fig. $4 C, D$, middle and right panels). Population averages within each group show that the most likely ISI of cells without a DAP is significantly shorter than that of cells with a DAP (4.12 \pm 0.12 vs $6.96 \pm 3.73 \mathrm{~ms}, p=0.01$, Kruskal-Wallis test); the same is true for the autocorrelation peaks $(4.13 \pm 0.11 \mathrm{vs} 9.46 \pm 4.41 \mathrm{~ms}$, $p=0.001$, Kruskal-Wallis test). These differences are readily explained by the different time courses of the post-spike voltage deflections: The rapid fAHP time course of $\mathrm{BD}^{+}$cells strongly reduces the chance that a second $\mathrm{AP}$ is fired directly after the first $\mathrm{AP}$, whereas in $\mathrm{BD}^{-}$cells the down-stroke of the first AP stops abruptly at depolarized levels, often above the AP threshold (Figs. $1 B, C, 4 A, B$ ).

In fact, the absence of an fAHP in $\mathrm{BD}^{-}$cells separates this group from $\mathrm{BD}^{+}$cells. Consistent with this picture, the fAHP increases the refractory period (taking the shortest $10 \%$ of the

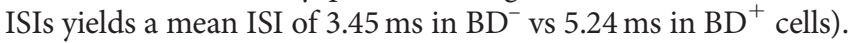
When the voltage dynamics are released from the fAHP, the DAP is uncovered, which opens a wide "window of opportunity" for a second $\mathrm{AP}$ in $\mathrm{BD}^{+}$cells. The resulting ISI distributions are broader for $\mathrm{BD}^{+}$cells $(6.7 \pm 3.27 \mathrm{~ms})$ compared with $\mathrm{BD}^{-}$cells (3.66 $\pm 0.36 \mathrm{~ms}, p=0.01$, Kruskal-Wallis test). For $\mathrm{BD}^{+}$cells, the most likely ISI in a burst mirrors $\Delta \mathrm{t}_{\mathrm{DAP}}$, the time interval between an AP and the succeeding DAP peak [median value $5.1 \mathrm{~ms}$, no difference with most likely ISI according to a median test $(p=0.61)$, which suggests a direct role of the post-AP dynamics in burst behavior.

The intrinsic voltage dynamics alone do not explain why sparsely-bursting cells have much broader ISI distributions (Fig. $4 D$ ). Other features correlated with SB behavior, however. All identified SB cells were pyramidal neurons, whereas only one out of four $\mathrm{BD}^{-}$cells were pyramidal; four of the five SB cells were in LIII, whereas $\mathrm{BD}^{-}$cells were solely found in LII. By contrast, the $\mathrm{BD}^{-}$and $\mathrm{BD}^{+}$groups were not distinguished by cell-type or the layer in which they were found, which makes it unlikely that anatomic differences can explain the observed variations in the spike trains of bursty neurons.

In vitro, stellate cells often produce spike doublets or brief bursts (Alessi et al., 2016). We tested whether spike trains in vivo showed similar preferences. For this purpose, we computed the frequency with which cells fired two, three, or more spikes in a burst. The frequency of bursts with exactly $n$ spikes decreases monotonically with $\mathrm{n}$, and does so for all three cell groups. Doublets and triplets were not overrepresented (Fig. 4E). In 21 out of the 24 cells, the distribution for $\mathrm{n}$ is consistent with an exponential distribution $\left(\chi^{2}\right.$ goodness of fit test for the correlation between linear fit and data after logarithmic transformation; $p>0.05)$. There is thus no preferred burst size or "unit of information," such as a spike doublet or triplet. In the spirit of hippocampal "complex spike bursts" (Ranck, 1973), a grid-cell burst can be regarded as just a sequence of two or more spikes with short ISIs. The exact choice of the cutoff threshold is not critical; qualitatively similar results were obtained using ISI thresholds from $8 \mathrm{~ms}$ up to $15 \mathrm{~ms}$ (data not shown).

\section{Spike-train characteristics of bursty cells are largely conserved across $1 \mathrm{D}$ and $2 \mathrm{D}$ environments}

So far, the analysis was based on a relatively small number of neurons recorded in head-fixed animals running in a virtual linear corridor (Domnisoru et al., 2013). To explore whether these data generalize to other experimental conditions, we analyzed a complementary dataset that contained 112 grid cells from mice that foraged at random in a square environment (Latuske et al., 2015). Although these extracellular recordings do not offer direct access to the membrane-potential dynamics, they might still reveal signatures of the different post-AP dynamics. In particular, we expected that grid cells would not only show the versus non-bursty dichotomy described by Latuske et al. (2015), but that there would also be qualitative differences within the bursty subpopulation.

To facilitate the comparison between the two datasets we kept the maximum lag of $50 \mathrm{~ms}$ and the 1-ms binning when analyzing spike-time autocorrelations. To minimize observer bias, $\mathrm{k}$-means cluster analyses were performed on the 50-dimensional raw autocorrelations as well as in principal-component spaces with $N=2-4$ dimensions. We analyzed the robustness of the k-means clustering results by calculating silhouette scores for each value of $\mathrm{k}$ (Rousseeuw, 1987). Irrespective of the dimensionality $\mathrm{N}$ of the data, separation into three clusters led to the best performance; the clusters hardly changed when using different numbers of principal components. Cluster assignment was stable, regardless of whether the autocorrelations were computed from the first or second half of a cell's spike train: $91.1 \%$ of the cells kept their cluster identity (Fig. $5 B$ ) from the first to the second half when using $N=3$ principal components. To compare these data with the previous results, we again plot the first two principal components against each other (Fig. 5A). The mean autocorrelation (Fig. 5C) closely resembled the one obtained from the virtual-track data (Fig. 3B); and so did the principal components (compare Figs. $5 D$ and $3 C$ ), as quantified by their similarity (the scalar product computed for time lags up to $50 \mathrm{~ms}$ ) of 0.86 for PC1 and 0.63 for PC2 when the two experimental conditions are compared.

There is also a high similarity between the corresponding group-averaged autocorrelations in both datasets (SB cells: 0.93, $\mathrm{BD}^{-}$cells: $0.93, \mathrm{BD}^{+}$cells: 094 ; compare Figs. $5 E$ and $4 C$ ). This is remarkable as the cluster analysis of the open-field data (Latuske et al., 2015) only reflects the overall structure of the grid-cell autocorrelations and is not informed by intracellular measurements. There is, however, one prominent difference between both datasets. The SB neurons recorded by Latuske et al. (Fig. $5 E$, left panel) fire hardly any spike within the first few milliseconds (so that the authors named them non-bursty neurons) and their autocorrelation has a pronounced peak at around $15 \mathrm{~ms}$. The autocorrelation function of the SB neurons recorded by Domnisoru et al. (2013; Fig. 4C, left panel) exhibits a local peak at around $4 \mathrm{~ms}$, and grows more slowly, with a local maximum at $30-40 \mathrm{~ms}$. On the other hand, the average autocorrelations of 


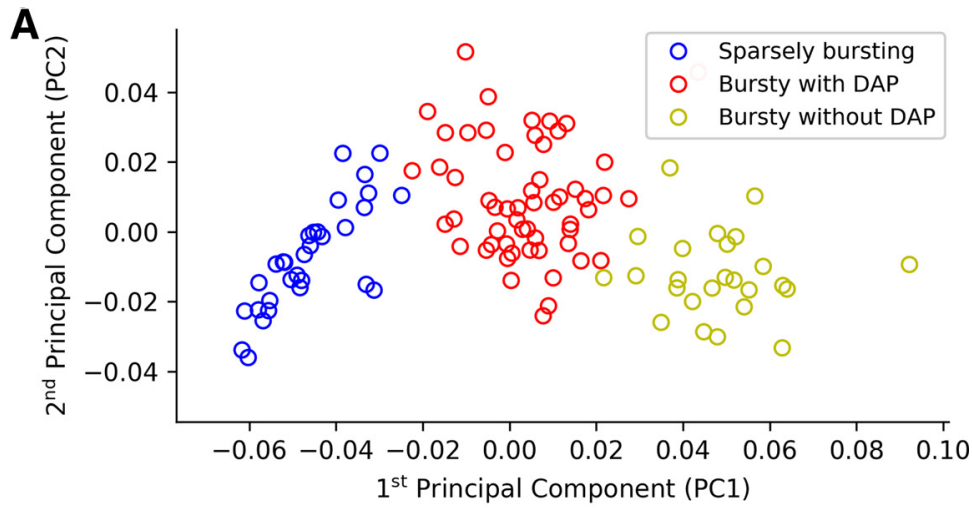

B
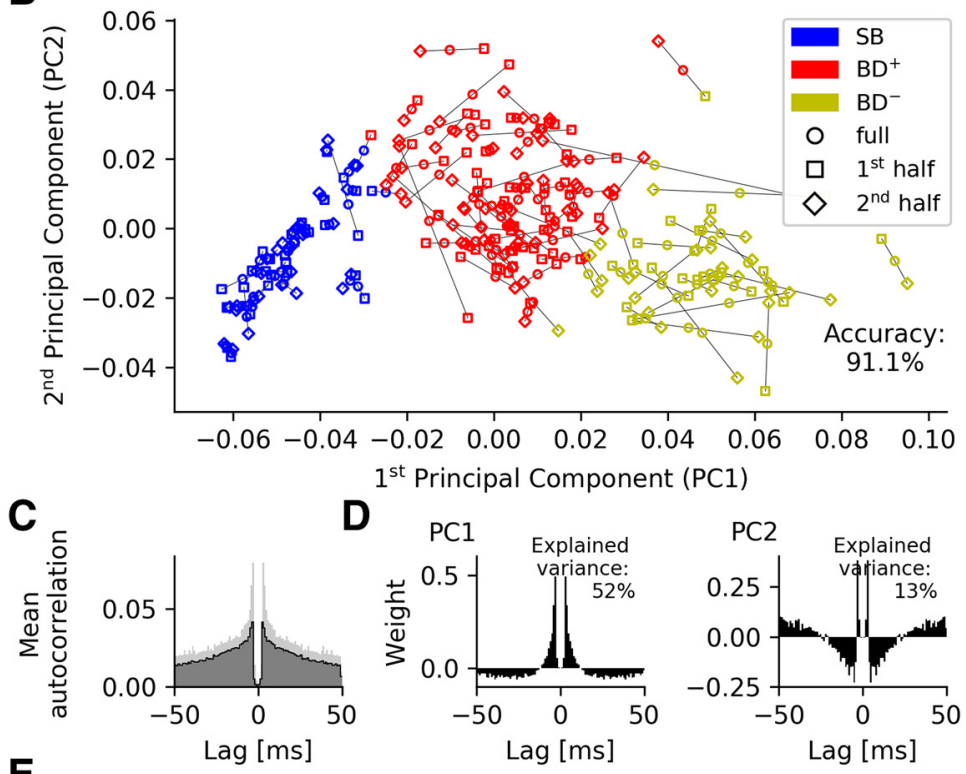

E
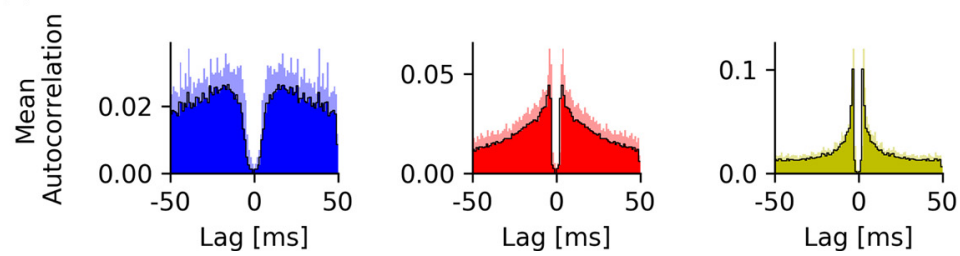

Figure 5. Spike-time autocorrelations of MEC grid cells from mice moving in open arenas. $\boldsymbol{A}$, k-means cluster analysis $(\mathrm{k}=3)$ of spike-time autocorrelations. $\boldsymbol{B}$, To test the robustness of the PCA-based class assignment in $\boldsymbol{A}$, we separately considered the first and second half of all spikes for each neuron. We then computed the autocorrelations within these two sets and projected the results into the $P C$ space of the full data. k-means clustering $(k=3)$ results in only $8.9 \%$ of neurons switching group identity, underscoring the robustness of the cluster analysis. $\boldsymbol{C}$, Mean autocorrelation across the grid cells. Shaded areas, SD. D, The first two principal components of the spike-time autocorrelations. The sharp peaks in $\boldsymbol{B}, \boldsymbol{C}$ again demonstrate the prevalence of short ISIs (here around $3.5 \mathrm{~ms}$ ) in the mean grid-cell discharge patterns as well as their cell-to-cell variability. Shaded areas, SD. $\boldsymbol{E}$, Autocorrelations averaged across all neurons from each cell group reveal a striking similarity between cells recorded on virtual tracks and in open fields. Strongest deviations are shown by non-bursting/SB cells in the Latuske et al. (2015) versus Domnisoru et al. (2013) data.

the $\mathrm{BD}^{+}$and $\mathrm{BD}^{-}$cells are almost identical when compared across both experimental conditions.

In the virtual-track data, the autocorrelations of the $\mathrm{BD}^{-}$neurons $(n=7)$ peak at $4.13 \mathrm{~ms}$ with a cell-to-cell variability of $0.11 \mathrm{~ms}$ (SD). The autocorrelations of the corresponding cells from the open-field recordings $(n=25)$ have their peak at $3.56 \mathrm{~ms}$ (SD: $0.27 \mathrm{~ms}$ ). The two experimental conditions also differ in the measured grid-field sizes: these are larger in virtual reality than open-field environments (Domnisoru et al., 2013, their supplemental Fig. 9). Consistent with this observation, other spike-train measures also reflect longer timescales in virtual versus open fields, e.g., the most likely ISI (Fig. 6A), the width of the ISI histogram (Fig. $6 B$ ) or the fraction of ISIs below $8 \mathrm{~ms}$ (Fig. 6C). Within each experimental setting, however, the three cell groups exhibited the same trends, albeit on slightly different timescales.

Differences between virtual-track and open-field data might be influenced by how spikes are measured intracellularly and extracellularly (Anastassiou et al., 2015). APs of BD neurons tend to slightly increase their width within a burst-like discharge pattern. This implies that the time interval between the peaks of two successive APs (i.e., the intracellularly measured ISI) can be somewhat longer than the time interval between the maximal upstroke slopes of the two APs, a proxy of the extracellularly measured ISI (Henze et al., 2000). Judging from the AP shapes of the $\mathrm{BD}^{-}$ neurons in the data of Domnisoru et al. (2013), this differential effect is, however, only on the order of $0.1-0.2 \mathrm{~ms}$ and thus cannot fully explain the observed timing differences. Other possible factors include differences in the animals' physiological state and stress level in these two highly different experimental conditions. The firing rates (Fig. 6D) are rather similar across experimental conditions and cell groups, which might reflect a general networklevel regulation of the average firing rate.

\section{Spatial response properties are similar across all three grid-cell groups}

In the next step of our analysis, we asked whether the pronounced differences between the temporal response characteristics of the three grid-cell groups translate into differences in their spatial firing patterns. The study of Latuske et al. (2015) had shown that this was not the case when one compares bursty with sparsely-bursting grid cells. However, the two groups of bursty neurons might still differ in their spatial behavior. To obtain reliable field estimates, we used the open-field data for this analysis. We tested several measures, including grid score (Sargolini et al., 2006), spatial information (Skaggs et al., 1996), and head-direction score (Sargolini et al., 2006), but could not detect any significant differences between $\mathrm{BD}^{+}$and $\mathrm{BD}^{-}$cells in these measures (Fig. 6E-G). Only the grid spacing (Hafting et al., 2005) shows a slight trend in that $\mathrm{BD}^{-}$cells had a somewhat smaller spacing (37.3 $6.6 \mathrm{~cm})$ than $\mathrm{BD}^{+}$cells $(41.6 \pm 6.9 \mathrm{~cm}$; Fig. $6 H)$. This finding is in agreement with results by Bant et al. (2020), who report that shorter intraburst ISIs go hand in hand with smaller grid periods. The effect was present, but not strong in the data presented here ( $p=0.019$, Kolmogorov-Smirnov test). Differences in grid spacing between bursting and SB cells $(38.7 \pm 6.3 \mathrm{~cm})$ were not significant ( $p=0.98$, Kolmogorov-Smirnov test). 
A
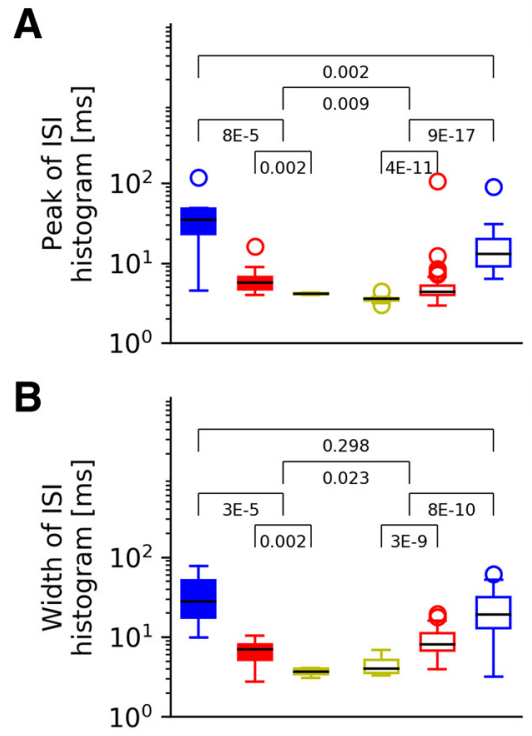

$$
\text { C }
$$

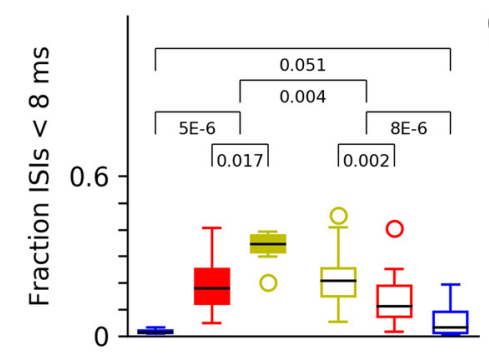

D

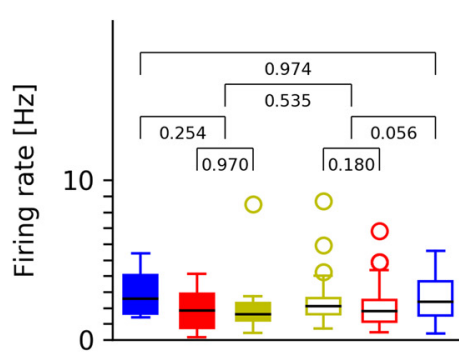

Temporal properties

$$
\begin{array}{r}
\text { Sparsely bursting } \\
\text { Bursty with DAP } \\
\text { Bursty without DAP }
\end{array}
$$$$
\text { Linear track }
$$$$
\text { (VR): }
$$

E

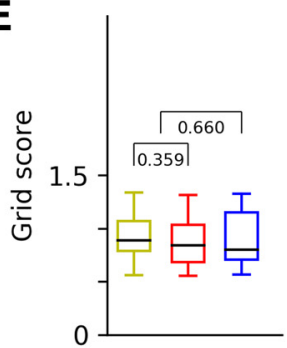

$\mathbf{F}$
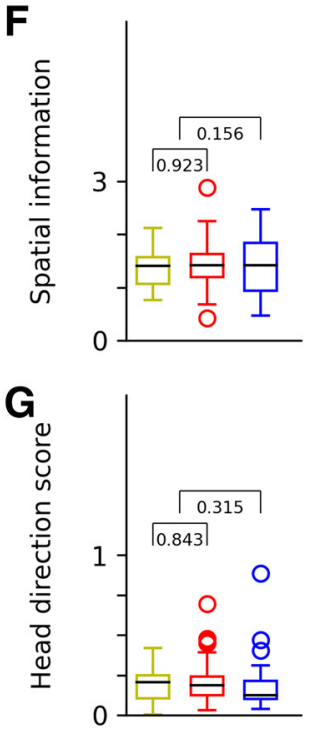

H

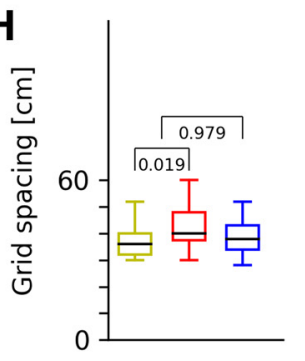

Spatial properties

Open

field:
Figure 6. Grid-cell spike-train characteristics across datasets and spatial coding. $\boldsymbol{A}-\boldsymbol{D}$, Comparison of linear-track data (filled symbols) and data from the open arena (un-filled symbols). $\boldsymbol{E}-\boldsymbol{G}$, Spatial properties of grid fields recorded in the open arena. $\boldsymbol{A}$, ISI peak, i.e., most likely ISI. B, Width of the ISI distribution. C, Fraction of ISIs below $8 \mathrm{~ms}$ ("burstiness"). $\boldsymbol{D}$, Mean firing rates. $\boldsymbol{E}$, Grid score. $\boldsymbol{F}$, Spatial information. $\boldsymbol{G}$, Head direction score. $\boldsymbol{H}$, Grid spacing. Despite strong differences in temporal spike-train characteristics $(\boldsymbol{A}-\boldsymbol{C})$, mean firing rates $(\boldsymbol{D})$ and spatial coding properties $(\boldsymbol{E}-\boldsymbol{H})$ of grid cells are largely conserved across all three cell groups, with a slight difference in grid spacing between $\mathrm{BD}^{+}$and $\mathrm{BD}^{-}$cells. The $p$ values are calculated from Kolmogorov-Smirnov tests.

Slice experiments show that DAPs of stellate cells can be modulated; if the holding potential is decreased, the amplitude $\Delta \mathrm{V}_{\mathrm{DAP}}$ of the following DAP increases, and it decreases whenever the holding potential is increased (Alessi et al., 2016). Domnisoru et al. (2013) found that a grid cell depolarizes as the animal crosses a firing field and hyperpolarizes in the out-of-field regions. Therefore, we wondered whether a $\mathrm{BD}^{+}$cell might preferentially generate DAP-mediated bursts on entering a grid field, as the hyperpolarization preceding the membrane-potential ramp might facilitate larger DAPs and thus make DAP-mediated burst firing in these neurons more likely.

To test this hypothesis, we took open-field data from Latuske et al. (2015) and investigated in detail whether spikes belonging to the bursts of a $\mathrm{BD}^{+}$cell had an above-chance probability to occur at the edges of its firing fields and, more generally, whether those spikes differed in their spatial statistics from other spikes of the same neuron, in the spirit of a place-cell study by Harris et al. (2001). In particular, we analyzed the distribution of spike distances from the respective firing-field centers as well as topological features of the discharge patterns of $\mathrm{BD}^{+}$cells, with special focus on ISIs expected for DAP-triggered bursts. For $\mathrm{BD}^{+}$cells, the field-wise mean distance between burst spikes and the respective firing-field center (normalized by each individual firingfield size to range between zero and unity) was $0.46 \pm 0.10$, significantly smaller than the same quantity evaluated for isolated spikes $\left(0.60 \pm 0.06, p=1.8 \times 10^{-4}\right.$ Kruskal-Wallis test). This shows that burst spikes are far more likely to occur near the firing-field center than isolated spikes and contradicts the hypothesis that burst spikes represent the edges of firing fields. The same was true for $\mathrm{BD}^{-}$cells $\left(0.44 \pm 0.06\right.$ vs $0.59 \pm 0.04, p=8.7 \times 10^{-5}$ Kruskal-Wallis test) and SB cells $(0.46 \pm 0.12$ vs $0.56 \pm 0.09$, $p=7.5 \times 10^{-3}$ Kruskal-Wallis test). Compared across the three cell groups, the mean distances for burst spikes were not statistically different ( $\mathrm{SB}$ vs $\mathrm{BD}^{+}: p=0.99, \mathrm{SB}_{\mathrm{Bs}} \mathrm{BD}^{-}: p=0.85, \mathrm{BD}^{+}$vs $\mathrm{BD}^{-}: p=0.72$, Kruskal-Wallis test). We thus we did not find a special role for bursts in spatial coding. As a complementary check, we used the data from Domnisoru et al. (2013) to search for whether DAP deflections measured in the firing fields of $\mathrm{BD}^{+}$cells were smaller than the DAP deflections of out-of-field spikes but did not find any obvious changes either.

These findings suggest that despite the striking differences in the spike-train patterns of bursty cells with and without DAP, these differences have no obvious consequences for the cells' spatial tuning properties. Temporal variations in the membrane potential, in particular the large $\theta$ oscillations observed in some bursty grid cells, are uncorrelated with the animal's trajectory and may easily mask less prominent spatial dependencies. In fact, decoupling of spatial and temporal tuning characteristics might endow the system with added plasticity and computational flexibility.

\section{Bursty grid cells: one continuum or two clusters?}

Since $\mathrm{BD}^{+}$and $\mathrm{BD}^{-}$cells showed indistinguishable spatial tuning, we reconsidered their partition into two distinct groups based on their temporal firing characteristics. Could it be that the data are better described as a single group with continuously varying parameters?

To answer this question, we went back to the grid-cell data from Domnisoru et al. (2013) and analyzed how the cells' salient spike-train characteristics depended on the two biophysical parameters $\Delta \mathrm{V}_{1}$ and $\Delta \mathrm{V}_{2}$ (Fig. 7). The mean firing rate does not correlate with $\Delta \mathrm{V}_{1}$ and $\Delta \mathrm{V}_{2}$ (Fig. $7 A, B$ ). There are also no significant dependencies within the $\mathrm{BD}^{+}$and $\mathrm{BD}^{-}$groups $\left(\Delta \mathrm{V}_{1}\right.$ : $p_{\mathrm{BD}+}=0.88, p_{\mathrm{BD}-}=0.24 ; \Delta \mathrm{V}_{2}: p_{\mathrm{BD}+}=0.58, p_{\mathrm{BD}-}=0.90$, as tested by independently shuffling the two coordinates of the respective data points and computing the Pearson correlation for each new sample; the $p$ value is given by the fraction of samples for which the correlation value was larger than in the original sample). 


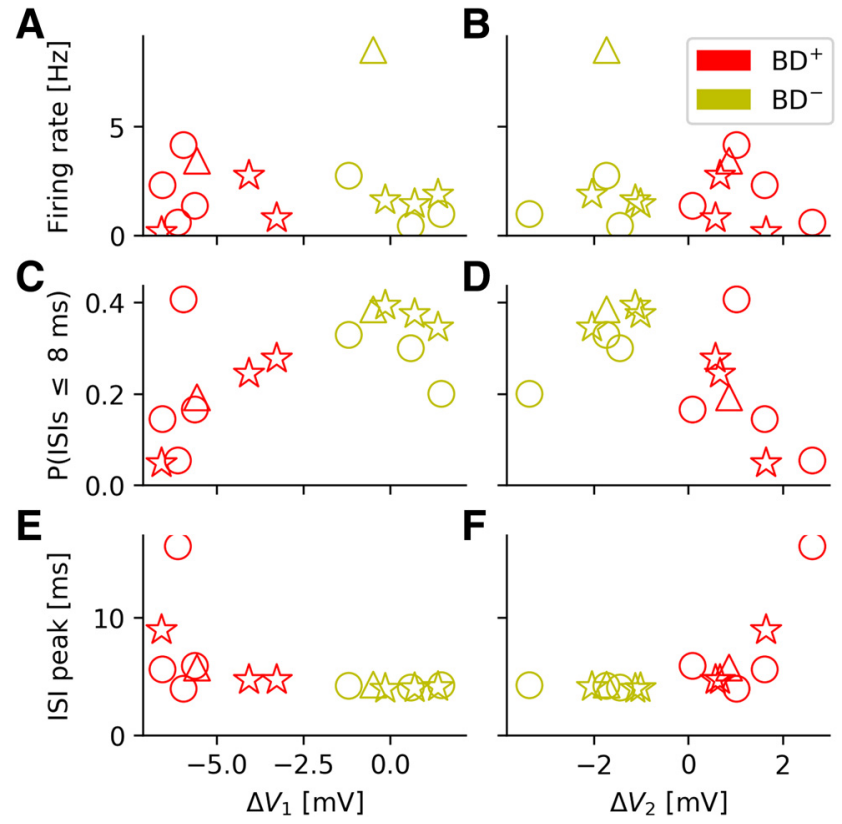

Figure 7. Cluster structure of the bursty-grid-cell population. Dependence of key spiketrain parameters on the amplitude of fast AHP $\left(\Delta \mathrm{V}_{1}\right)$ and afterdepolarization $\left(\Delta \mathrm{V}_{2}\right)$. For a definition of these two parameters, see, e.g., the caption of Figure 1. $A, B$, mean firing rates. C, $\boldsymbol{D}$, Fraction of ISls below $8 \mathrm{~ms}$ ("burstiness"). Across the entire population of bursty neurons, the larger $\Delta \mathrm{V}_{1}$, the more frequent are short ISIs. $E, F$, Location of ISI peak. While the firing rates do not exhibit a trend, neither within the two cell groups nor across the groups, the other quantities depicted show trends that differ from the null-hypothesis (no increase/ decrease as a function of $\Delta \mathrm{V}_{1}$ or $\Delta \mathrm{V}_{2}$ ). The data also suggest that the population of bursty neurons either forms one joint although under-sampled cloud or contains two distinct subpopulations. In either case the spike-train characteristics do depend on the cells' DAP properties.

A different picture emerges when the fraction of short ISIs (below $8 \mathrm{~ms}$ ) is considered (Fig. 7C,D). Visual inspection suggests a joint trend for $\mathrm{BD}^{+}$and $\mathrm{BD}^{-}$cells; the larger $\Delta \mathrm{V}_{1}$, the more frequent are short ISIs (Fig. 7C). With a $p=0.013$, this trend is statistically significantly different from the null-hypothesis (no increase/decrease as a function of $\Delta \mathrm{V}_{1}$ ), and in agreement with our earlier functional interpretation of DAPs: for negative $\Delta \mathrm{V}_{1}$ (i.e., $\mathrm{BD}^{+}$cells), cells quickly hyperpolarize, making very short ISIs rare.

Consistent with this observation, the location of the ISI peak tends to grow for increasingly negative $\Delta \mathrm{V}_{1}$ (Fig. $7 E$ ) and increasingly positive $\Delta \mathrm{V}_{2}$ (Fig. $7 F$ ) if the entire population of bursty neurons is considered $\left(\Delta \mathrm{V}_{1}: p=0.04, \Delta \mathrm{V}_{2}: p=0.01\right)$. Within the $\mathrm{BD}^{-}$population, however, the ISI peak does hardly vary at all, as emphasized before.

These results indicate that there is no clear-cut answer to the question whether the population of bursty neurons forms one joint although under-sampled cloud or contains two distinct subpopulations. More importantly, however, is the observation that in either case, certain spike-train characteristics do depend on the cells' individual DAP properties, which supports the view that DAPs do not only exist under in vivo conditions but may also play a functional rule.

\section{Non-grid cells show same DAP and spike-train characteristics as grid cells}

In the last step of our analysis, we asked whether non-grid cells differed from grid cells in their DAP behavior or spike-time autocorrelation characteristics. To this end, we first determined
A
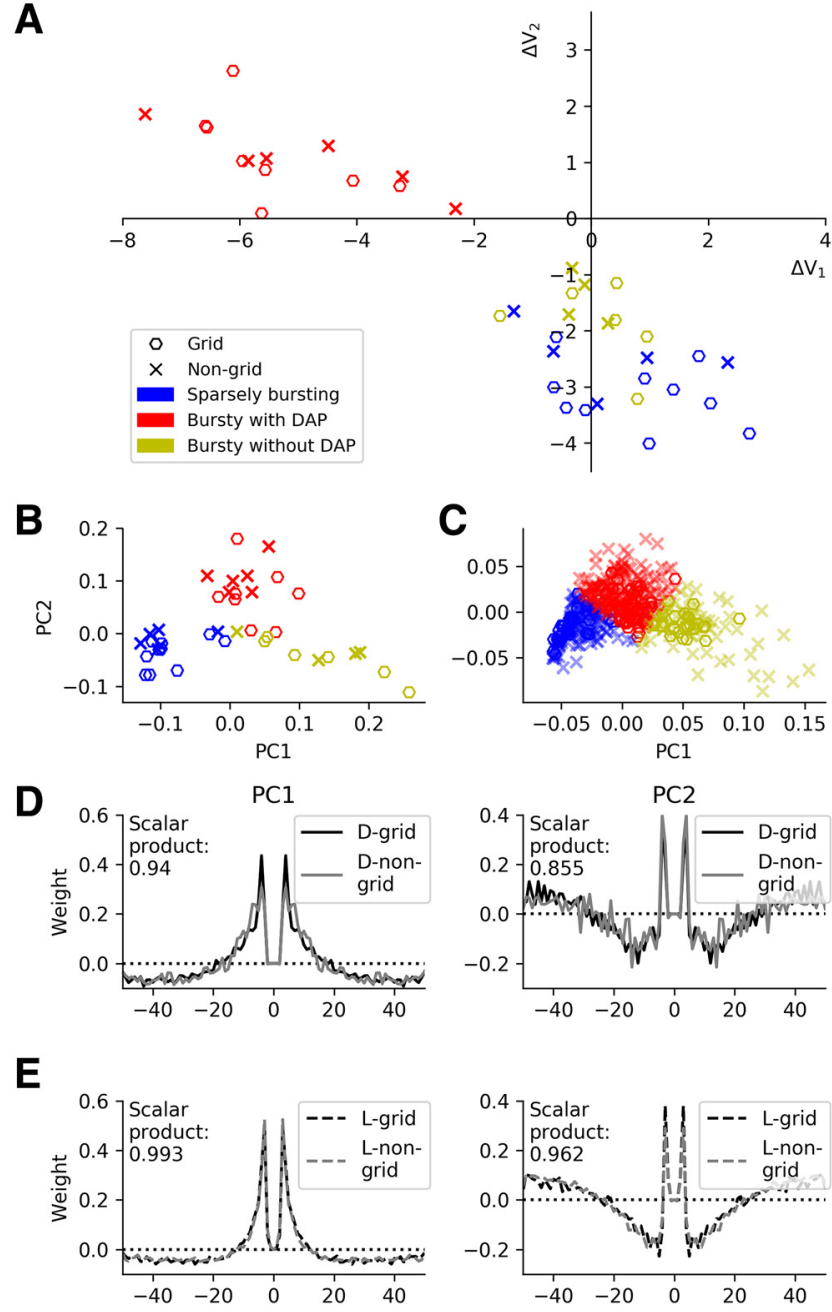

Figure 8. Comparison of the characteristics of grid cells and non-grid cells. $A$, Quantification of spike afterpotentials as in Figure $1 D$ but now for all principal cells recorded on the virtual linear track. $\boldsymbol{B}, \mathrm{PCA}$ of spike-time autocorrelations as in Figure $3 A$ but now for all principal cells recorded on the virtual linear track. C, PCA of spike-time autocorrelations as in Figure $5 A$ but now for all principal cells recorded in the open environment. The high similarity between grid cells and non-grid cells suggests that the three functional cell classes identified in this study are universal across all principal cells in the superficial MEC layers. $\mathbf{D}$; First and second principal components (left and right panels, respectively) of the virtual-track dataset for grid cells and non-grid cells. The similarity of the $P C$ components is measured by the scalar product for time lags up to $50 \mathrm{~ms}$. $\boldsymbol{E}$, As in $\boldsymbol{D}$, but now for the data recorded in the open environment. The values of the scalar products are rather close to their maximal value of 1 and underscore the similarity of the grid-cell and non-grid-cell autocorrelations and their cluster structure.

the DAP parameters $\Delta \mathrm{V}_{1}$ and $\Delta \mathrm{V}_{2}$ for all 40 neurons on linear tracks in virtual reality. As shown in Figure 8A, non-grid cells (represented by " $\mathrm{x}$ ") fall into the same data clouds as the grid cells (represented by open hexagons) when these two intracellular measures are considered. Similarly, a PCA of the spike-train autocorrelations of the entire dataset (Fig. $8 B$ ) exhibits a 2D structure that is highly reminiscent of that when only grid cells are taken into account (Fig. 3A). The same is true for the PCA of the spike-train autocorrelations of the open-field data from Latuske et al. (2015), shown in Figure 8C. In fact, even the principal components themselves are highly similar when computed for grid cells only or for non-grid cells only (Fig. 8D,E). Moreover, the entire dataset exhibits the same ambiguity concerning the "one versus two clusters" question (Fig. 9) as when only grid cells are considered (Fig. 7). 


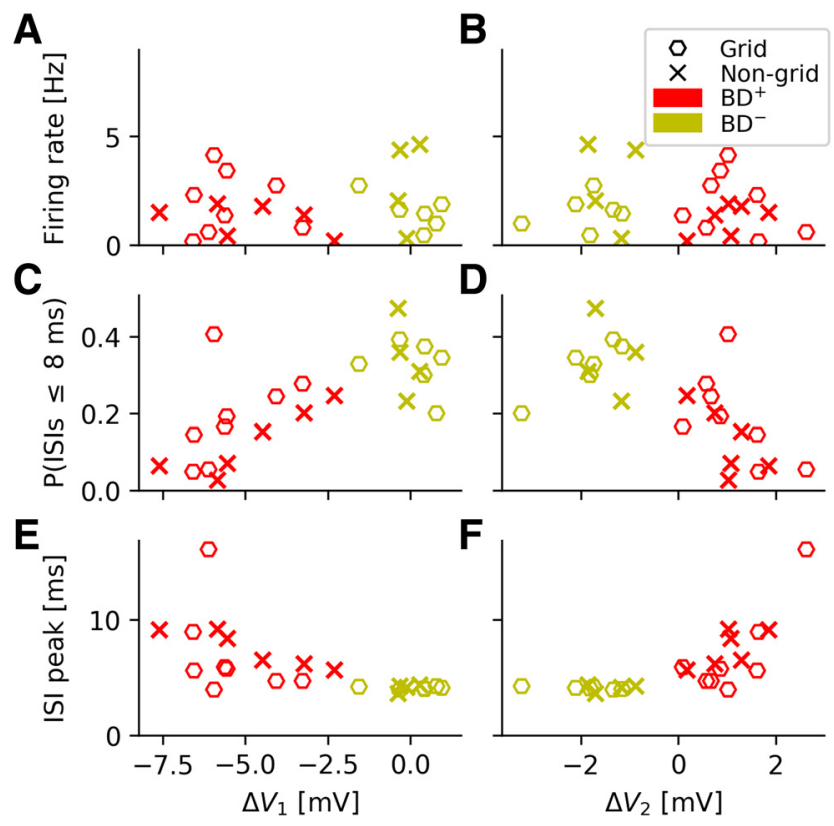

Figure 9. Cluster structure of all bursty principal cells recorded in virtual reality. Dependence of key spike-train parameters on fast AHP $\left(\Delta \mathrm{V}_{1}\right)$ and afterdepolarization $\left(\Delta \mathrm{V}_{2}\right)$. $A, B$, Mean firing rates. $C, D$, Fraction of ISIs below $8 \mathrm{~ms}$ ("burstiness"). $E$, $\boldsymbol{F}$, Location of ISI peak. There is no apparent difference to the grid-cell cluster structure shown in Figure 7.

Consistent with this observation, the remarkably small cellto-cell variability in the autocorrelation peaks of $\mathrm{BD}^{-}$grid cells (virtual linear track: $4.13 \pm 0.11 \mathrm{~ms}$; open field: $3.56 \pm 0.27 \mathrm{~ms}$ ) is also shared by the non-grid $\mathrm{BD}^{-}$cells (virtual linear track: $4.05 \pm 0.25 \mathrm{~ms}$, open field: $3.67 \pm 0.60 \mathrm{~ms}$ ), and these peaks are significantly shorter than the autocorrelation peaks of the nongrid $\mathrm{BD}^{+}$cells (virtual linear track: $9.29 \pm 3.53 \mathrm{~ms}, p=0.02$, Kruskal-Wallis test; open field: $6.99 \pm 3.52 \mathrm{~ms}, p=1.15 \times 10^{-29}$, Kruskal-Wallis test). All data: mean values \pm SD. The SD describes the cell-to-cell variability.

Taken together, our findings demonstrate not only that the DAP characteristics of grid cells have no consequences for their spatial firing properties (Fig. $6 E-G$ ) but that in addition, nongrid cells and grid cells fall into the same three subgroups, SB neurons, bursty with DAP and bursty without DAP. Both results suggest that DAPs and burst firing are not critical for spatial navigation.

\section{Discussion}

Tetrode recordings in freely moving rats (Mizuseki et al., 2009; Ebbesen et al., 2016) and mice (Latuske et al., 2015) have shown that principal neurons in superficial MEC layers come in two functional subclasses, cells that burst frequently and others that do not or only rarely burst. Our analysis of whole-cell data from mice running on linear virtual tracks (Domnisoru et al., 2013) suggests that principal cells located in Layer III tend to be SB and that they do not generate DAPs, in agreement with previous slice studies in rats (Canto and Witter, 2012).

Bursty neurons varied strongly in the overall shape of their autocorrelations (as shown for grid cells in Fig. 4C) and their ISI distributions (Fig. 4D). This diversity can be understood in terms of the cell-specific shapes of spike afterpotentials: Neurons without a DAP (BD ${ }^{-}$cells) had ISI distributions that peaked sharply at around $4 \mathrm{~ms}$ and varied only minimally across that group of cells whereas the ISIs of neurons with a DAP $\left(\mathrm{BD}^{+}\right.$cells) were most frequent between 5 and $15 \mathrm{~ms}$.
At first sight, the gap between $\mathrm{BD}^{+}$and $\mathrm{BD}^{-}$cells in the $\Delta \mathrm{V}_{1}-\Delta \mathrm{V}_{2}$ diagram (Fig. $1 D$ ) speaks against a continuum of bursty grid cells and rather points to the existence of two separate subgroups. This impression might, however, be due to a sampling artifact; there are only 15 such cells with intracellular recordings in the dataset from Domnisoru et al. (2013). We, therefore, investigated the dependencies of various spike-train characteristics on $\Delta V_{1}$ and $\Delta V_{2}$ (Fig. 7). The smooth behavior of some measures, such as the burstiness, i.e., the fraction of ISIs below $8 \mathrm{~ms}$, or the ISI-peak location, and the lack of any sharp transitions in the other measures, support the characterization of the $\mathrm{BD}^{-}$and $\mathrm{BD}^{-}$grid-cell subgroups as a single, although sparsely populated group that spans a range of properties. The $\mathrm{BD}^{+}$and $\mathrm{BD}^{-}$grid-cell subgroups have a roughly equal stellateto-pyramidal-cell ratio $(3: 1)$, which means that cellular morphology is not indicative of the cell's type of bursting behavior. Moreover, the bursting behavior of non-grid cells is almost identical (Fig. $8 A$ ) to grid cells (see also Fig. $8 D, E$ ), so the spatial encoding properties of neurons are also not an indicator of their bursting behavior.

Consistent with the hypothesis of one single group of bursty neurons, The physiological properties of individual cells could either be fixed or undergo plastic changes that move the biophysical cell parameters between the $\mathrm{BD}^{+}$and $\mathrm{BD}^{-}$regions. In the $\Delta \mathrm{V}_{1}-\Delta \mathrm{V}_{2}$ space (Figs. $1 D, 8 \mathrm{~A}$ ), a transition from $\mathrm{BD}^{+}$to $\mathrm{BD}^{-}$ corresponds to an increase in $\Delta \mathrm{V}_{1}$ accompanied by a somewhat smaller decrease in $\Delta V_{2}$. Such a parameter change can be achieved through modifications of the AP-threshold, fAHP minimum and/or DAP maximum, as illustrated by the arrows in Figure 2A. Various ion channels have been implicated in DAP generation, from sodium and calcium channels (Alessi et al., 2016), to potassium (Eder et al., 1991) and HCN channels (Dickson et al., 2000), which also play a key role for slower gridcell rhythms (Giocomo and Hasselmo, 2009). These channels could be regulated, e.g., by cholinergic stimulation, which has been shown to induce DAPs and after discharges in MEC-LayerII neurons (Magistretti et al., 2004). Such modulations would have a direct impact on the precise temporal characteristics of bursting neurons. In addition, cells with $\mathrm{BD}^{-}$characteristics and thus shorter intraburst ISIs seem to have smaller grid spacing than $\mathrm{BD}^{+}$cells, in accordance with findings by Bant et al. (2020), although the significance of our results is weak.

Modulations of the biophysical parameters governing the afterpotentials might even occur at the time scale of single runs through the animal's environment. Indeed, close inspection of individual membrane-potential traces suggests that $\mathrm{BD}^{+}$cells do not generate a DAP after every AP; conversely, some APs of $\mathrm{BD}^{-}$ cells are followed by a DAP. One might even speculate that most bursty cells are capable of generating DAPs, slice experiments in rats suggest $85 \%$ of Layer-II stellate cells and $73 \%$ of Layer-II pyramids have DAPs (Canto and Witter, 2012), but that this mechanism is under external control so as to switch cells between $\mathrm{BD}^{+}$ and $\mathrm{BD}^{-}$behavior.

Remarkably, the ISI distributions of $\mathrm{BD}^{-}$cells have ultrasharp peaks, whose location varies only minimally within that group. Notably, the same short ISIs are shown by the SB neurons in Layer III (see also Mizuseki et al., 2009) and could be elicited if Layer-III SB neurons received (convergent) synaptic input from Layer-II $\mathrm{BD}^{-}$cells. The precise function of burst sequences in the $250-$ to $300-\mathrm{Hz}$ regime remains an open question. Similarly, it is not obvious how cells with highly distinct firing characteristics can be orchestrated to create one joint grid-cell network (but see Pastoll et al., 2013), in which the SB, $\mathrm{BD}^{-}$, and 
$\mathrm{BD}^{+}$cell classes have roughly the same grid score, spatial information and head direction score (Fig. $5 E-G$ ). With their high rate of bursts, $\mathrm{BD}^{-}$neurons might be ideally suited to drive other neurons in the network, whereas the DAPs of $\mathrm{BD}^{+}$cells might trigger synaptic plasticity, similar to their function in CA3 pyramidal neurons (Mishra et al., 2016), and thus play a critical role for network reconfiguration when the animal learns about new environments (Krupic et al., 2018) or goals (Boccara et al., 2019).

Switching on the DAP mechanism (without interfering with the preceding fAHP) would then increase the probability of additional APs (Alessi et al., 2016) as well as provide a trace for the long-term potentiation of incoming synapses (Mishra et al., 2016). Once these synapses are strengthened and the DAP mechanism has been turned off again (or masked), the cell can fire precisely tuned bursts with short ISIs. These cell-intrinsic processes could be complemented by precisely wired and timed synaptic inputs (Varga et al., 2010; Couey et al., 2013; Pastoll et al., 2013; Buetfering et al., 2014; Fuchs et al., 2016; Schmidt et al., 2017; Winterer et al., 2017). Through short-term plasticity and integrative postsynaptic processes (Lisman, 1997; Izhikevich et al., 2003) such reorganization could result in a stronger influence on downstream neurons.

In contrast to what one might have expected, the strong dependence of DAPs on the neuron's recent history (Alonso and Klink, 1993; Canto and Witter, 2012; Alessi et al., 2016) does not seem to translate into a spatial burst code. For example, one might have hypothesized that the DAP of a Layer-II stellate cell should be particularly large when the animal is moving into one of the cell's firing fields, as this corresponds to raising the membrane potential from its previous out-of-field hyperpolarization. However, we could not find any signature for the ring-like burstfield structure expected in this scenario. In fact, we could not find any nontrivial spatial dependencies despite vigorous search. This came as a surprise, given the role of burst firing for spatial coding in the hippocampus (Harris et al., 2001) or subiculum (Simonnet and Brecht, 2019). Similarly, spike doublets do not seem to play any special role for burst coding. Together, these findings suggest that grid-cell bursts are either not used for spatial coding, apart from their contribution to $\theta$-phase precession (Hafting et al., 2008; Reifenstein et al., 2012), or that the spatial coding is masked by temporal fluctuations that are uncorrelated with spatial coordinates.

It is well known that after-spike potentials play a critical role in the control of AP firing patterns. For example, mAHPs control $\theta$-band clustering of APs in MEC stellate neurons (Fransén et al., 2004). Our study extends these and related findings to the $250-$ to $300-\mathrm{Hz}$ range and provides a novel mechanistic explanation of MEC burst firing. Given the inherent limitations of headfixed virtual reality experiments, whole-cell recordings from rotationally unconstrained animals, such as performed by Chen et al. (2019), should provide further insight into the mechanisms underlying burst phenomena in rodent MEC.

Acknowledgements: We thank D. W. Tank and K. Allen for making data from Domnisoru et al. (2013) and Latuske et al. (2015), respectively, available; K. Thurley for support in statistical analysis; and K. Allen, C. Domnisoru, and S. Häusler for stimulating discussions and helpful feedback on the manuscript.

\section{References}

Alessi C, Raspanti A, Magistretti J (2016) Two distinct types of depolarizing afterpotentials are differentially expressed in stellate and pyramidal-like neurons of entorhinal-cortex layer II. Hippocampus 26:380-404.
Alonso A, Klink R (1993) Differential electroresponsiveness of stellate and pyramidal-like cells of medial entorhinal cortex layer II. J Neurophysiol 70:128-143.

Anastassiou CA, Perin R, Buzsáki G, Markram H, Koch C (2015) Cell typeand activity-dependent extracellular correlates of intracellular spiking. J Neurophysiol 114:608-625.

Bant JS, Hardcastle K, Ocko SA, Giocomo LM (2020) Topography in the bursting dynamics of entorhinal neurons. Cell Rep 30:2349-2359.

Boccara CN, Nardin M, Stella F, O’Neill J, Csicsvari J (2019) The entorhinal cognitive map is attracted to goals. Science 363:1443-1447.

Buetfering C, Allen K, Monyer H (2014) Parvalbumin interneurons provide grid cell-driven recurrent inhibition in the medial entorhinal cortex. Nat Neurosci 17:710-718.

Canto CB, Witter MP (2012) Cellular properties of principal neurons in the rat entorhinal cortex. II. The medial entorhinal cortex. Hippocampus 22:1277-1299.

Chen G, Lu Y, King JA, Cacucci F, Burgess N (2019) Differential influences of environment and self-motion on place and grid cell firing. Nat Commun 10:630.

Chrobak JJ, Buzsáki G (1998) Gamma oscillations in the entorhinal cortex of the freely behaving rat. J Neurosci 18:388-398.

Colgin LL, Denninger T, Fyhn M, Hafting T, Bonnevie T, Jensen O, Moser MB, Moser EI (2009) Frequency of gamma oscillations routes flow of information in the hippocampus. Nature 462:353-357.

Couey JJ, Witoelar A, Zhang SJ, Zheng K, Ye J, Dunn B, Czajkowski R, Moser MB, Moser EI, Roudi Y, Witter MP (2013) Recurrent inhibitory circuitry as a mechanism for grid formation. Nat Neurosci 16:318-324.

Dickson CT, Magistretti J, Shalinsky MH, Fransén E, Hasselmo ME, Alonso A (2000) Properties and role of I_h in the pacing of subthreshold oscillations in entorhinal cortex layer II neurons. J Neurophysiol 83:2562-2579.

Domnisoru C, Kinkhabwala AA, Tank DW (2013) Membrane potential dynamics of grid cells. Nature 495:199-204.

Ebbesen CL, Reifenstein ET, Tang Q, Burgalossi A, Ray S, Schreiber S, Kempter R, Brecht M (2016) Cell type-specific differences in spike timing and spike shape in the rat parasubiculum and superficial medial entorhinal cortex. Cell Rep 16:1005-1011.

Eder C, Ficker E, Gündel J, Heinemann U (1991) Outward currents in rat entorhinal cortex stellate cells studied with conventional and perforated patch recordings. Eur J Neurosci 3:1271-1280.

Engel TA, Schimansky-Geier L, Herz AVM, Schreiber S, Erchova I (2008) Subthreshold membrane-potential resonances shape spike-train patterns in the entorhinal cortex. J Neurophysiol 100:1576-1589.

Fransén E, Alonso AA, Dickson CT, Magistretti J, Hasselmo ME (2004) Ionic mechanisms in the generation of subthreshold oscillations and action potential clustering in entorhinal layer II stellate neurons. Hippocampus 14:368-384.

Fuchs EC, Neitz A, Pinna R, Melzer S, Caputi A, Monyer H (2016) Local and distant input controlling excitation in layer II of the medial entorhinal cortex. Neuron 89:194-208.

Giocomo LM, Hasselmo ME (2009) Knock-out of HCN1 subunit flattens dorsal-ventral frequency gradient of medial entorhinal neurons in adult mice. J Neurosci 29:7625-7630.

Hafting T, Fyhn M, Bonnevie T, Moser M-B, Moser EI (2008) Hippocampus-independent phase precession in entorhinal grid cells.

Hafting T, Fyhn M, Molden S, Moser M-B, Moser EI (2005) Microstructure of a spatial map in the entorhinal cortex. Nature 436:801-806.

Harris KD, Hirase H, Leinekugel X, Henze DA, Buzsáki G (2001) Temporal interaction between single spikes and complex spike bursts in hippocampal pyramidal cells. Neuron 32:141-149.

Hasselmo ME (2014) Neuronal rebound spiking, resonance frequency and theta cycle skipping may contribute to grid cell firing in medial entorhinal cortex. Philos Trans R Soc Lond B Biol Sci 369:20120523.

Henze DA, Borhegyi Z, Csicsvari J, Mamiya A, Harris KA, Buzsáki G (2000) Intracellular features predicted by extracellular recordings in the hippocampus in vivo. J Neurophysiol 84:390-400.

Izhikevich EM, Desai NS, Walcott EC, Hoppensteadt FC (2003) Bursts as a unit of neural information: selective communication via resonance. Trends Neurosci 26:161-167.

Krupic J, Bauza M, Burton S, O'Keefe J (2018) Local transformations of the hippocampal cognitive map. Science 359:1143-1146. 
Latuske P, Toader O, Allen K (2015) Interspike intervals reveal functionally distinct cell populations in the medial entorhinal cortex. J Neurosci 35:10963-10976.

Lisman JE (1997) Bursts as a unit of neural information: making unreliable synapses reliable. Trends Neurosci 20:38-43.

Magistretti J, Ma L, Shalinsky MH, Lin W, Klink R, Alonso A (2004) Spike patterning by $\mathrm{Ca}^{2+}$-dependent regulation of a muscarinic cation current in entorhinal cortex layer II neurons. J Neurophysiol 92:1644-1657.

Mishra RK, Kim S, Guzman SJ, Jonas P (2016) Symmetric spike timing-dependent plasticity at CA3-CA3 synapses optimizes storage and recall in autoassociative networks. Nat Commun 7:11552.

Mizuseki K, Sirota A, Pastalkova E, Buzsáki G (2009) Theta oscillations provide temporal windows for local circuit computation in the entorhinalhippocampal loop. Neuron 64:267-280.

Newman EL, Hasselmo ME (2014) Grid cell firing properties vary as a function of theta phase locking preferences in the rat medial entorhinal cortex. Front Syst Neurosci 8:193.

Pastoll H, Solanka L, van Rossum MC, Nolan MF (2013) Feedback inhibition enables $\theta$-nested $\gamma$ oscillations and grid firing fields. Neuron 77:141-154.

Pedregosa F, Varoquaux G, Gramfort A, Michel V, Thirion B, Grisel O, Blondel M, Prettenhofer P, Weiss R, Dubourg V, VanderPlas J, Passos A, Cournapeau D, Brucher M, Perrot M, Duchesnay V (2011) Scikit-learn: machine learning in Python. J Mach Learn Res 12:2825-2830.

Ranck JBJ (1973) Studies on single neurons in dorsal hippocampal formation and septum in unrestrained rats. I. Behavioral correlates and firing repertoires. Exp Neurol 41:461-531.

Reifenstein ET, Kempter R, Schreiber S, Stemmler MB, Herz AVM (2012) Grid cells in rat entorhinal cortex encode physical space with independent firing fields and phase precession at the single-trial level. Proc Natl Acad Sci USA 109:6301-6306.

Rousseeuw PJ (1987) Silhouettes: a graphical aid to the interpretation and validation of cluster analysis. Comput Appl Math 20:53-65.

Sargolini F, Fyhn M, Hafting T, McNaughton BL, Witter MP, Moser M-B, Moser EI (2006) Conjunctive representation of position, direction, and velocity in entorhinal cortex. Science 312:758-762.

Schmidt H, Gour A, Straehle J, Boergens KM, Brecht M, Helmstaedter M (2017) Axonal synapse sorting in medial entorhinal cortex. Nature 549:469-475

Schmidt-Hieber C, Häusser M (2013) Cellular mechanisms of spatial navigation in the medial entorhinal cortex. Nat Neurosci 16:325-331.

Simonnet J, Brecht M (2019) Burst firing and spatial coding in subicular principal cells. J Neurosci 39:3651-3662.

Skaggs WE, McNaughton BL, Wilson MA, Barnes CA (1996) Theta phase precession in hippocampal neuronal populations and the compression of temporal sequences. Hippocampus 6:149-172.

Varga C, Lee SY, Soltesz I (2010) Target-selective GABAergic control of entorhinal cortex output. Nat Neurosci 13:822-824.

Virtanen P, Gommers R, Oliphant TE, Haberland M, Reddy T, Cournapeau D, Burovski E, Peterson P, Weckesser W, Bright J, van der Walt SJ, Brett M, Wilson J, Millman KJ, Mayorov N, Nelson ARJ, Jones E, Kern R, Larson E, Carey CJ, et al. (2020) SciPy 1.0: fundamental algorithms for scientific computing in Python. Nat Methods 17:261-272.

Winterer J, Maier N, Wozny C, Beed P, Breustedt J, Evangelista R, Peng Y, D'Albis T, Kempter R, Schmitz D (2017) Excitatory microcircuits within superficial layers of the medial entorhinal cortex. Cell Rep 19:1110-1116. 